OPEN ACCESS

Edited by:

Jean Celli,

Washington State University, USA

Reviewed by:

Janakiram Seshu,

University of Texas at San Antonio,

USA

Edgardo Moreno, National University of Costa Rica,

Costa Rica

${ }^{*}$ Correspondence:

Anne Keriel

anne.kerie@@inserm.fr

Received: 21 July 2016 Accepted: 15 September 2016 Published: 28 September 2016

Citation:

Soler-Lloréns PF, Quance CR, Lawhon SD, Stuber TP, Edwards JF,

Ficht TA, Robbe-Austerman S, O'Callaghan D and Keriel A (2016) A Brucella spp. Isolate from a Pac-Man

Frog (Ceratophrys ornata) Reveals

Characteristics Departing from

Classical Brucellae.

Front. Cell. Infect. Microbiol. 6:116.

doi: 10.3389/fcimb.2016.00116

\section{A Brucella spp. Isolate from a Pac-Man Frog (Ceratophrys ornata) Reveals Characteristics Departing from Classical Brucellae}

\author{
Pedro F. Soler-Lloréns ${ }^{1,2}$, Chris R. Quance ${ }^{3}$, Sara D. Lawhon ${ }^{4}$, Tod P. Stuber ${ }^{3}$, \\ John F. Edwards ${ }^{4}$, Thomas A. Ficht ${ }^{5}$, Suelee Robbe-Austerman ${ }^{3}$, David O'Callaghan ${ }^{1,2}$ \\ and Anne Keriel ${ }^{1,2 *}$ \\ ${ }^{1}$ Institut National de la Santé et de la Recherche Médicale, U1047, UFR de Médecine, Nimes, France, ${ }^{2}$ Université de \\ Montpellier, U1047, Nimes, France, ${ }^{3}$ Mycobacteria and Brucella Section, National Veterinary Services Laboratories, Animal \\ and Plant Health Inspection Service, United States Department of Agriculture, Ames, IA, USA, ${ }^{4}$ Clinical Microbiology, \\ Department of Veterinary Pathobiology, College of Veterinary Medicine and Biomedical Science, Texas A\&M University, \\ College Station, TX, USA, ${ }^{5}$ Department of Veterinary Pathobiology, Texas A\&M University, College Station, TX, USA
}

Brucella are highly infectious bacterial pathogens responsible for brucellosis, a frequent worldwide zoonosis. The Brucella genus has recently expanded from 6 to 11 species, all of which were associated with mammals; The natural host range recently expanded to amphibians after some reports of atypical strains from frogs. Here we describe the first in depth phenotypic and genetic characterization of a Brucella strains isolated from a frog. Strain B13-0095 was isolated from a Pac-Man frog (Ceratophyrus ornate) at a veterinary hospital in Texas and was initially misidentified as Ochrobactrum anthropi. We found that B13-0095 belongs to a group of early-diverging brucellae that includes Brucella inopinata strain $\mathrm{BO} 1$ and the $\mathrm{B}$. inopinata-like strain $\mathrm{BO} 2$, with traits that depart significantly from those of the "classical" Brucella spp. Analysis of B13-0095 genome sequence revealed several specific features that suggest that this isolate represents an intermediate between a soil associated ancestor and the host adapted "classical" species. Like strain BO2, B13-0095 does not possess the genes required to produce the perosamine based LPS found in classical Brucella, but has a set of genes that could encode a rhamnose based O-antigen. Despite this, B13-0095 has a very fast intracellular replication rate in both epithelial cells and macrophages. Finally, another major finding in this study is the bacterial motility observed for strains $\mathrm{B} 13-0095, \mathrm{BO} 1$, and $\mathrm{BO} 2$, which is remarkable for this bacterial genus. This study thus highlights several novel characteristics in strains belonging to an emerging group within the Brucella genus. Accurate identification tools for such atypical Brucella isolates and careful evaluation of their zoonotic potential, are urgently required.

Keywords: Brucella, frog, motility, metabolism, LPS, virulence, evolution, Ochrobactrum 


\section{INTRODUCTION}

Brucella are highly infectious bacterial pathogens responsible for brucellosis, a frequent worldwide zoonosis $(500,000$ new cases/year). Infection is usually transmitted to humans by direct contact, ingestion of contaminated animal products or aerosolization (De Figueiredo et al., 2015). The disease is mainly characterized by abortion or sterility in animals, while in humans it is a serious febrile disease with varied symptomatology including undulant fever, headaches, muscle pain, night sweats, asthenia, and depression (Moreno, 2014). Infection often requires hospitalization and at least 6 weeks of antibiotic treatment; if left untreated, patients can develop serious complications such as neurobrucellosis or endocarditis.

Brucella are Gram-negative coccobacilli belonging to the family brucellaceae together with Ochrobactrum spp., their closest phylogenetic relatives. This family belongs to the order Rhizobiales, which contain several genera of plant-associated alpha proteobacteria. The Brucella genus is currently composed of 11 species, some of them considered to be pathogenic to humans. The classification of the six "historical" species is based on phenotypic characteristics and each has a preferential host: Brucella melitensis (goats), Brucella suis (suidae), Brucella abortus (cows), Brucella ovis (sheep), Brucella canis (dogs), and Brucella neotomae (desert rats) (Moreno, 2014). Over the past few decades strains isolated from marine mammals have been included in the genus as Brucella ceti (cetaceae) and Brucella pinnipedialis (pinnipeds) (Foster et al., 2007). The most recently identified species are Brucella microti (voles, Audic et al., 2009), Brucella inopinata (a human breast implant infection, Scholz et al., 2010), Brucella papionis (stillbirth in baboons, Whatmore et al., 2014), and Brucella vulpis (red foxes, Scholz et al., 2016). Other atypical Brucella-like strains have not been assigned a specific designation; $B$. inopinata-like strain $\mathrm{BO} 2$ (chronic pneumonia in a human) (Tiller et al., 2010a) and strains isolated from wild rodents in Australia (Tiller et al., 2010b). Isolation of Brucella strains from non-mammalian hosts is exceptional, however three reports recently described the isolation of atypical Brucella strains from amphibians: a Big-eyed tree frog (Leptopelis verniculatus, Fischer et al., 2012), several African bullfrogs (Pyxicephalus edulis, Eisenberg et al., 2012) and a White's tree frog (Litoria caerulea, Whatmore et al., 2015).

Brucella are facultative intracellular pathogens that can evade the innate defense mechanisms and reach an intracellular replication niche comprised of vesicles derived from the endoplasmic reticulum (Celli, 2015). Intracellular replication of Brucella requires a type IV secretion system (T4SS), an apparatus used by many bacterial pathogens to deliver effectors into host cells and that is considered as a major virulence factor in Brucella (Celli, 2006; Lacerda et al., 2013). It also depends on the chemical structure of their lipopolysaccharide (LPS, Porte et al., 2003; Mancilla, 2016). LPS is composed of an O-polysaccharide (OPS, or O-antigen), a long chain formed by one or two sugar repeats, lipid A, which anchors LPS in the outer membrane, and a short core oligosaccharide (OS) that serves as a linker between lipid A and O-PS. Other than two naturally occurring rough species (B. canis and B. ovis) there is little variability in the composition and structure of the LPS within the Brucella genus (Lapaque et al., 2005). Exceptions to this rule were described for the recently isolated $B$. inopinata strain $\mathrm{BO} 1$ and strains isolated from Australian wild rodents, which appear to have modification in the LPS core, and for strain $\mathrm{BO} 2$ which produces an atypical O-PS (Wattam et al., 2012; Zygmunt et al., 2012).

Here, we report the first case of Brucella infection of an amphibian in America, confirming that these Brucella are found throughout the world. This strain (B13-0095) was isolated from a Pac-Man frog (Ceratophyrus ornate) in a veterinary hospital in Texas. It was initially misidentified as Ochrobactrum anthropi, but belongs to an emerging clade of atypical Brucella that includes the strains $\mathrm{BO} 1$ and $\mathrm{BO} 2$ ("BO clade"). Analysis of the B13-0095 genome led to the identification of new metabolic pathways shared with $O$. anthropi, several specific to B13-0095, and others that are conserved within the $\mathrm{BO}$ clade. We also found that this strain produces and atypical O-PS and has a faster intracellular replication rate than classical Brucella spp. Finally, we demonstrate that the BO clade Brucella strains exhibit motility, a phenotype not described for this bacterial genus.

\section{MATERIALS AND METHODS}

\section{Bacterial Strains}

Bacterial strains used in this study are listed in Table $\mathbf{1 .}$ Upon primary isolation and characterization, the Pac-Man frog Brucella isolate was cultured onto trypticase soy (TS) agar plates supplemented with $5 \%(\mathrm{v} / \mathrm{v})$ sheep's blood (BAP) (Becton Dickinson; Franklin Lakes, NJ) and incubated at $37^{\circ} \mathrm{C}$ in an atmosphere supplemented with $5 \%(\mathrm{v} / \mathrm{v})$ carbon dioxide. Slants of agar media (Becton Dickinson; Franklin Lakes, NJ) inoculated with the organism were incubated overnight at $37^{\circ} \mathrm{C}$ in an air atmosphere. For further experiments, all strains were cultured on TS medium at $37^{\circ} \mathrm{C}$. Fluorescent derivatives of the Pac-Man frog isolate (bIN1996) or of B. suis 1330 (bIN1505) were obtained by electroporation with pMR10 based plasmid encoding mCherry.

\section{Brucella-Specific PCR}

PCR was performed using primers Bruc-F (5'-AACCACGCT TGCCTTGCACACC- $3^{\prime}$ ) and Bruc-R (5'TTTCAAGCGCCT GTTCACCCG-3') (Scholz et al., 2008a). The PCR reaction included $2.5 \mathrm{mM} \mathrm{MgCl}_{2}, 2.0 \mathrm{mM}$ dNTPs, $150 \mathrm{nM}$ of each primer, and $0.5 \mathrm{U}$ AmpliTaq DNA Polymerase (Applied Biosystems; Foster City, CA).

\section{Genome Analysis}

Whole genome sequencing (WGS) was performed as described recently (Quance et al., 2016). Phylogenetic placement was performed using kSNP, a program that can analyze raw, unassembled reads (Gardner and Hall, 2013) using the genomes listed in Table S1. Genome assembly and automatic annotation was performed using the PATRIC resource ${ }^{1}$ (Wattam et al., 2014a). Confirmation of the presence of predicted protein encoding genes in other genomes was performed using BLASTP ${ }^{2}$ within the Brucella genus (Taxonomic ID 234) or Brucellaceae

\footnotetext{
${ }^{1}$ https://www.patricbrc.org

${ }^{2}$ http://blast.ncbi.nlm.nih.gov
} 
TABLE 1 | List of bacterial strains used in this study.

\begin{tabular}{|c|c|}
\hline Strain name & Description (reference) \\
\hline B13-0095 & Brucella isolate from a Pac-Man frog (this study) \\
\hline 1330 & Brucella suis biovar 1 type strain, ATCC $23444^{\top}$ \\
\hline $1330 \Delta$ VirB8 & $\Delta$ VirB8 mutant in B. suis 1330 (Paschos et al., 2006) \\
\hline $16 \mathrm{M}$ & Brucella melitensis biovar 1 type strain, ATCC $23456^{\top}$ \\
\hline 2308 & Brucella abortus biovar 1 \\
\hline BO1 & Brucella inopinata type strain, isolated from an infected breast implant in a 71-year-old patient from Oregon, USA (Scholz et al., 2010) \\
\hline $\mathrm{BO} 2$ & B. inopinata-like isolate, isolated from a 52-year-old Australian patient with chronic destructive pneumonia (Tiller et al., 2010a) \\
\hline CCM4915 & Brucella microti strain BCCN 07-01; isolated from infected voles in the Czech Republic (Scholz et al., 2008b) \\
\hline NF2653 & Representative of atypical Brucella strains isolated from wild rats in Queensland, Australia (Tiller et al., 2010b) \\
\hline O. anthropi ATCC49188 & Ochrobactrum anthropi type strain \\
\hline
\end{tabular}

family (Taxonomic ID 118882). A gene was considered to be present and conserved when the identity of the protein sequences were $\geq 90 \%$ within the Brucella genus. When comparing Brucella with Ochrobactrum we used $\geq 75 \%$ over $\geq 95 \%$ of the sequence as a cut off for presence of a homologe.

\section{Use of Different Carbon Sources}

Bacteria were grown in a chemically defined medium as previously described (Barbier et al., 2014). Briefly, bacteria were pre-cultured in a modified Plommet medium containing erythritol $(2 \mathrm{~g} / \mathrm{L})$ for $16 \mathrm{~h}$, washed with PBS and adjusted to an $\mathrm{OD}_{600}$ of 0.02 in modified Plommet containing either erythritol, ectoine $(20 \mathrm{mM})$, or L-rhamnose $(1 \mathrm{~g} / \mathrm{L})$. Growth was measured by reading the $\mathrm{OD}_{600}$ after $24 \mathrm{~h}$ of culture.

\section{Motility Assays}

In tube motility assays were performed in Difco ${ }^{\mathrm{TM}}$ Motility GI Medium (Becton Dickinson) supplemented with $0.05 \mathrm{~g} / \mathrm{L} \mathrm{2,3,5-}$ triphenyltetrazolium chloride (TTC) to help visualization of motility, according to the manufacturers recommendations. On plate swimming assays were performed as previously described (Ha et al., 2014). Briefly, TS plates containing 0.25\% (w/v) agar were inoculated with a $5 \mu \mathrm{l}$ drop of bacterial suspension at an $\mathrm{OD}_{600}$ of 1 at their center and motility was followed macroscopically for up to 5 days. Each condition was tested in independent experiments at least three times. Motility surface was measured using Image $\mathrm{J}^{3}$.

\section{LPS Analysis}

Extraction of LPS with SDS-proteinase $\mathrm{K}$ was performed as previously described (Soler-Lloréns et al., 2014). Extracts were separated on $11 \%(\mathrm{w} / \mathrm{v})$ polyacrylamide gels and stained using the periodate-alkaline silver method (Tsai and Frasch, 1982). The reaction was stopped with $1 \%(\mathrm{v} / \mathrm{v})$ acetic acid and pictures were taken with a Panasonic Lumix DMZ-6 camera.

\section{Intracellular Replication Profiles}

Human epithelial (HeLa) and murine macrophage cells (J774) were infected using gentamycin protection assays with an MOI of 500 or 50, respectively as described previously (Keriel et al., 2015).

${ }^{3}$ http://imagej.net/
TABLE 2 | $\mathrm{API}^{\circledR} 20 \mathrm{NE}$ Biochemical test results at 24 and $48 \mathrm{~h}$ for the Pac-Man frog Brucella isolate B13-0095.

\begin{tabular}{lll}
\hline Test & $24 \mathrm{~h}$ & $48 \mathrm{~h}$
\end{tabular}

Reduction of nitrates to nitrites or nitrogen

Indole production

Fermentation of glucose

Arginine dihydrolase

Urease

Hydrolysis of esculin

Hydrolysis of gelatin

$\beta$-galactosidase production

Glucose assimilation

Arabinose assimilation

Mannose assimilation

Mannitol assimilation

$\mathrm{N}$-acetyl-glucosamine assimilation

Maltose assimilation

Potassium gluconate assimilation

Capric acid assimilation

Adipic acid assimilation

Malate assimilation

Trisodium citrate assimilation

Phenyl acetic acid assimilation

Cytochrome oxidase

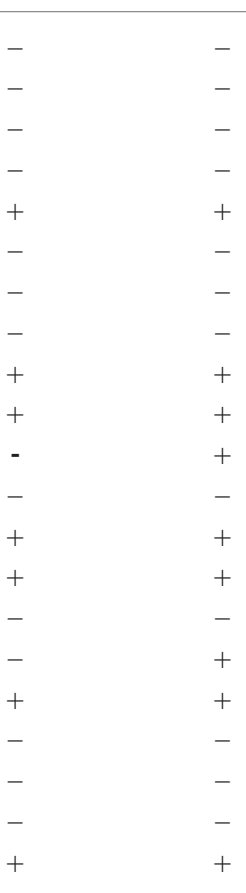

At $2,5,24$, and $48 \mathrm{~h}$ post-infection (hpi), cells were lysed with $0.1 \%$ Triton X-100 (v/v) for $10 \mathrm{~min}$. Serial dilutions of the cell lysates were then plated on TS plates and colonies were counted after 1 or 2 days of incubation at $37^{\circ} \mathrm{C}$.

\section{Microscopy Analysis}

Cells were plated on sterile glass coverslips, infected as described above and fixed with paraformaldehyde (3\% (w/v) PFA, 25 min, RT) at 24 hpi. Slides were mounted using ProLong gold media (Invitrogen) and visualized using a confocal microscope (Fluoview Fv10i, Olympus). For low magnification visualization, non-confocal images were acquired at a $1024 \times 1024$ resolution using a 10x objective (NA 0.4). Confocal images were acquired 
with a $1024 \times 1024$ resolution using the XY mode of the confocal microscope and a 60x oil immersion objective (NA 1.35).

\section{Flow Cytometry Analysis}

Cells were infected as above with the exception that mCherryexpressing bacteria were used. At 24 hpi cells were detached with trypsin, fixed, and analyzed by flow cytometry (MACSQuant VYB, Miltenyi). Data were analyzed using the MACSQuantify software. This gave $\%$ of infected cells (i.e., \% of mCherry positive cells) as well as the MFI (median fluorescence intensity) of infected cells, which is indicative of the number of bacteria inside cells.

\section{RESULTS}

\section{Case Report}

A six-year-old female, $192 \mathrm{~g}$, reticulated Pac-Man frog (Ceratophrys ornata) was presented to the Texas A\&M University Veterinary Medical Teaching Hospital (College Station, TX, USA) in February 2010 for depression, anorexia, weight loss, and a history of ingestion of foreign bodies (animal ID: 191570).

Five months prior to presentation, the frog ingested small glass beads that were part of the decorations in her enclosure, and was then moved to a different enclosure that contained a moss substrate. The following month, the frog ate an adult mouse and passed normal feces. Subsequently, the frog did not eat, despite the multiple attempts to feed her, and did not pass feces. The owner reported that the live prey used to feed the frog traumatized its digits and back. Prior to ingesting the foreign bodies, the frog had no history of illness. This pet was fed with a diet of live mice and received no additional nutritional supplements. A natural sunlight lamp was used to illuminate the frog's enclosure, and it was covered at night with a towel. The ambient temperature of the enclosure was maintained between 21$24^{\circ} \mathrm{C}$.

On presentation, the frog was unresponsive with fixed and dilated pupils. No pupillary light response was noted. Three to five, glass beads were palpable within the coelomic cavity. The distal phalanges of digits 3 and 4 of the right rear leg were missing, and skin over the back was eroded. The frog was determined to be $5-10 \%$ dehydrated. No other apparent abnormalities were noted on physical exam. The frog had a body condition score of 1 out of 5 . Because of a poor prognosis for recovery, and after informed consent of the owner, the frog was humanely euthanized by first anesthetizing it with a tricaine methanesulfonate (MS-222) bath followed by intracoelomic, pentobarbital sodium injection. Euthanasia was confirmed via Doppler ultrasound.

At necropsy, the liver was tan and friable, and the coelomic cavity of the frog had numerous strands of fibrin and firm adhesions adhering the coelomic organs to each other and to the body wall indicating subacute to chronic peritonitis. The stomach contained six, $2 \times 1 \mathrm{~cm}$, elliptical, smooth-surfaced glass beads, but grossly, they did not appear to have affected the stomach. No evidence of metabolic bone disease was observed. No significant lesions were observed in the other body systems. Histologic examination of the liver indicated severe, diffuse, vacuolar degeneration with intrahistiocytic pigment. The final diagnosis was severe, chronic peritonitis. Samples of the liver and peritoneum were submitted for bacterial culture.

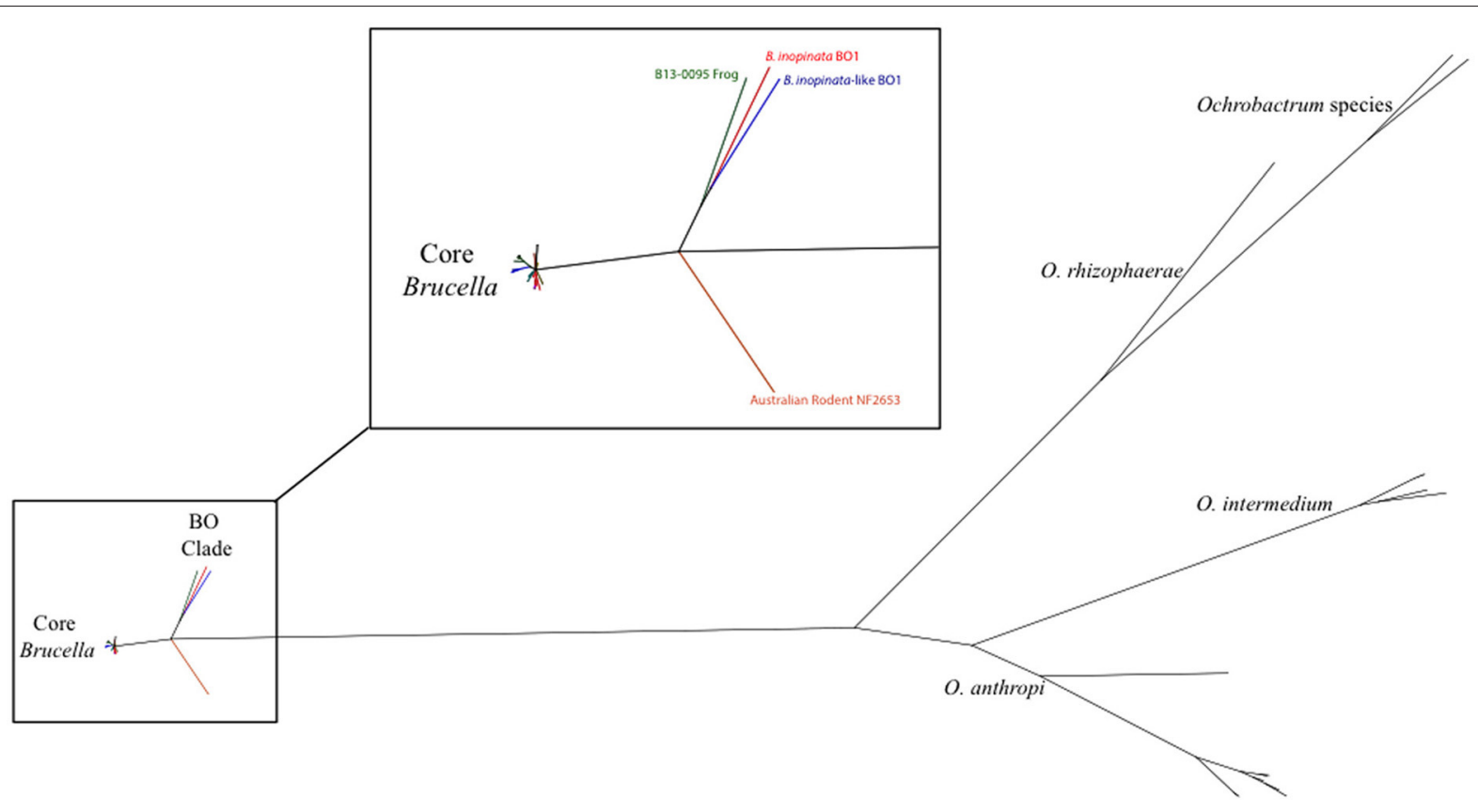

FIGURE 1 | Phylogenetic placement of B13-0095 relative to other Brucella spp. and Ochrobactrum spp. determined by kSNP. The reference strains for all classical Brucella spp. and novel species were included. The magnified region highlights the divergence point of the "BO clade" in Brucella. 


\section{Bacterial Isolation, Phenotypic Characterization and Identification}

The liver and peritoneum specimens were inoculated onto culture plates. Following overnight incubation, pinpoint, alphahemolytic bacterial colonies grew from the liver (culture 10060005-1), and peritoneum (culture 10606004-1). Slants of triple sugar iron (TSI) agar, lysine iron agar (LIA), urea agar, Simmons' citrate agar and tryptophan broth were inoculated with the organism. The organism was oxidase and urease positive, and did not utilize citrate or produce indole from tryptophan. On the TSI and LIA slants, the isolate did not ferment lactose or sucrose and did not produce hydrogen sulfide.

An API 20NE identification kit (bioMérieux) performed on the peritoneum isolate yielded identification codes of 0241324 at $24 \mathrm{~h}$ and 0243334 at $48 \mathrm{~h}$ (Table 2). When compared with the API online database (https://apiweb.biomerieux.com), this isolate was identified as $O$. anthropi with a probability of 99.9\%. However, some results were not consistent with this identification such as the positive adipic acid assimilation, the negative malate assimilation and the absence of nitrate reduction at $48 \mathrm{~h}$. Literature searches were performed at PubMed using various combinations of the search terms of "Ochrobactrum," "Brucella," "frog," and "amphibian." No case reports indicating prior isolation of Brucella species from frogs were found so no further efforts to identify the organism were made. Because of the unusual nature of the case, the isolates were stored in $10 \%$ glycerol at $-80^{\circ} \mathrm{C}$.

Several years after the original isolation of the organism, the literature search was repeated and yielded a report of isolation of a potentially novel Brucella species from frogs (Eisenberg et al., 2012) along with a case report documenting abscesses associated with a $B$. inopinata-like bacterium in a big-eyed tree frog purchased from a pet store (Fischer et al., 2012). The two isolates were thus revived and a $r e c A$ gene-based multi-primer PCR was performed. Both yielded a 167 bp product, specific to the Brucella genus (data not shown). An attempt was made to characterize the liver isolate (thereafter referred as B13-0095) using traditional Brucella biochemical analysis (Alton et al., 1988) at the National Veterinary Services Laboratories (NVSL, Ames, IA, USA). The isolate did not conform to the characteristics of any recognized Brucella species. The unusual profile included strong urease activity, no requirement for carbon dioxide, no production of hydrogen sulfide, no sensitivity to the dyes thionin or basic fuchsin at 1:25,000, no agglutination with monospecific anti-A and $M$ serum, and no lysis by Tbilisi phage at RTD or RTD $\times 10^{4}$. B13-0095 was later analyzed by matrix-assisted laser desorption/ionization time-of-flight (MALDI-TOF) mass spectrometry, using a Vitek MS and a database able to identify Brucella isolates at the species level (J. Mesureur and A. Keriel, unpublished data). B13-0095 was clearly discriminated from $O$. anthropi and was identified as $B$. inopinata with an identification score $\geq 99.99 \%$.

B13-0095 has an atypical growth rate for Brucella, as it gives 2-3 mm large colonies within $48 \mathrm{~h}$ at $37^{\circ} \mathrm{C}$ on blood-agar plates. We quantified its growth rate in liquid broth, compared to $B$. suis 1330 as a representative of classical Brucella species. B13-0095 reached stationary phase within $5 \mathrm{~h}$ of culture, whereas B. suis required at least $24 \mathrm{~h}$ to do so (data not shown), confirming that B13-0095 has a very rapid growth in vitro.

\section{Analysis of the Pac-Man Frog Isolate Genome}

Whole genome sequencing (WGS) was performed on B130095 at the NVSL. Analysis shows the presence of the IS711 insertion, a genetic hallmark of Brucella. A phylogenetic tree was drawn using kSNP (Gardner and Hall, 2013) with "core" Brucella spp., comprising the classically described pathogenic species, and some recently described "atypical" Brucella strains (Figure 1). B13-0095 clearly fell within a clade containing the $B$. inopinata strain $\mathrm{BO} 1$ and the $B$. inopinata-like isolate $\mathrm{BO} 2$ that have been previously described (Wattam et al., 2012). This group, further referred as the $\mathrm{BO}$ clade, was very close to strain NF2653, a representative of Australian wild rodents isolates, which is consistent with previous findings (Wattam et al., 2012). This clade is also less distant from Ochrobactrum than the classical Brucella spp. Full sequence alignment of the $16 \mathrm{~S}$ rRNA gene of B13-0095 with sequences of $\mathrm{BO} 1, \mathrm{BO} 2$, the Brucella spp. consensus sequence and O. anthropi showed that B13-0095 shares $100 \%$ sequence identity to those of $\mathrm{BO} 1$ and $\mathrm{BO} 2,99.6 \%$

TABLE 3 | List of protein encoding genes found to be specific the Brucella strain B13-0095 among the sequenced Brucella genomes using a FigFam search in PATRIC. ${ }^{4}$

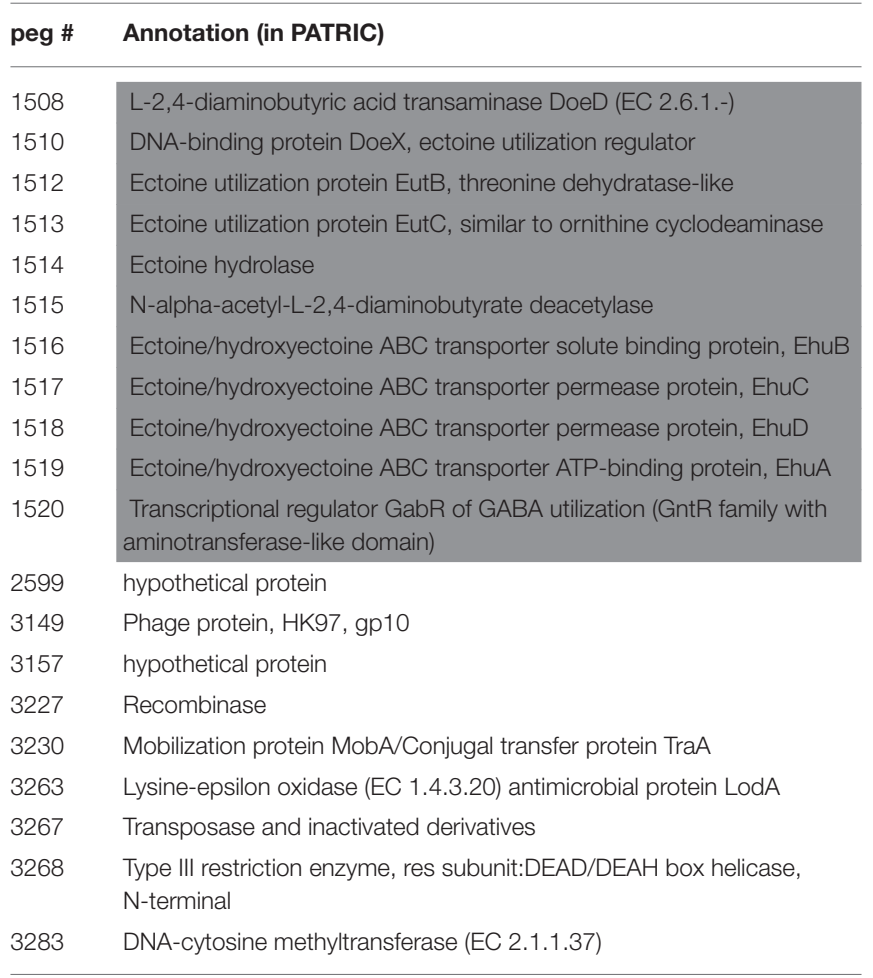

The genes highlighted in gray belong to the ectoine utilization cluster described in Figure 2A.

${ }^{4}$ https://www.patricbrc.org 
A

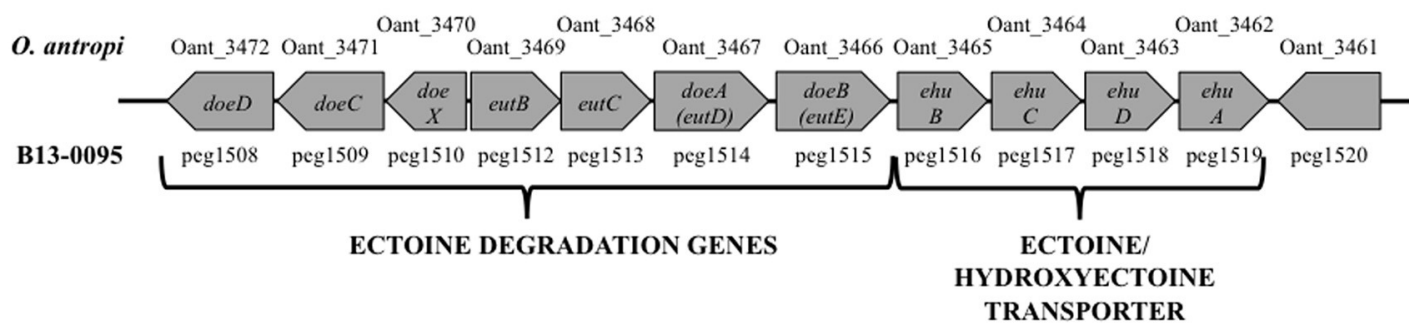

B

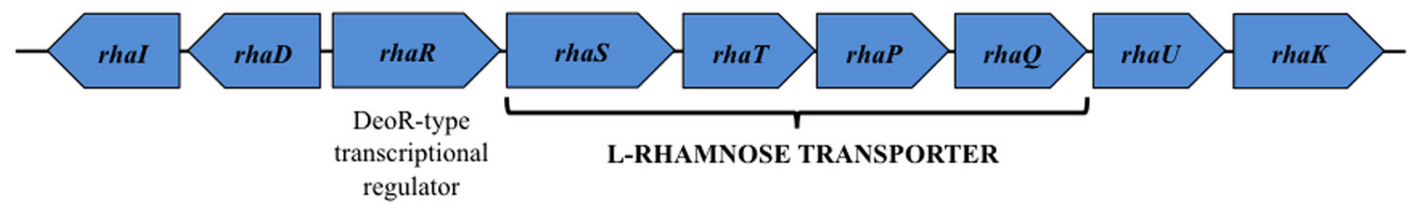

C

O. anthropi

Flg. locus

Flg. locus II

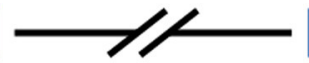

Rham

B13-0095, BO1 \& BO2

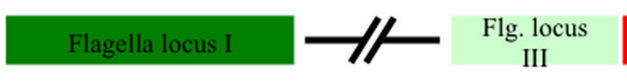

Flg. locus II

Classical Brucella
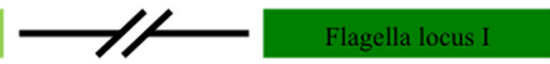

FIGURE 2 | Genetic clusters specific to B13-0095, BO1, and BO2. (A) Schematic representation of a $12.5 \mathrm{~kb}$ ectoine utilization genetic cluster conserved in B13-0095 and O. anthropi. (B) Schematic representation of the genes organization in a cluster involved in rhamnose utilization that is conserved in the B13-0095, $\mathrm{BO1}, \mathrm{BO}$, and $\mathrm{O}$. anthropi. (C) Position of the cluster of rhamnose utilization (in blue) relatively to the three loci of flagella related genes (in green) in the BO clade Brucella, compared to $\mathrm{O}$. anthropi and classical Brucella. The red box represents a fragment of $14 \mathrm{~kb}$ containing genes involved in several metabolic pathways or cationic antimicrobials resistance that is present in all Brucella.

identity with other Brucella spp. and $97.7 \%$ with $O$. anthropi ATCC 49188. The draft sequence was deposited in NCBI under the accession number SAMN05277611. The FASTQ files were deposited in NCBI Short Read Archive under the bioproject number PRJNA326393.

B13-0095 genome was then assembled, resulting in 38 contigs (338 bp to $767 \mathrm{kbp}$ ). Automatic annotation using RAST predicted 3326 protein encoding genes (peg), and PATRIC FigFam search identified 20 that seem unique to B13-0095 among the Brucella genus (Table 3) and 26 that are conserved within the BO clade, among which 10 are also present in $O$. anthropi genome (Table S2). Notably, the genes of the virB operon, which encode the components of Brucella T4SS, are present in the genome of B130095 (from peg152 to peg142, annotated as VirB1 and VirB11, respectively).

\section{An Ectoine Catabolic Pathway Unique to the Pac-Man Frog Isolate among the Brucella Genus}

Among the unique features in B13-0095 genome, we found that a $12.5 \mathrm{~kb}$ fragment encompassing 11 genes highly conserved between B13-0095 and O. anthropi ATCC49188. Most of
TABLE 4 | Carbon sources in Brucella sp. B13-0095, BO1, and BO2.

\begin{tabular}{lcccc}
\hline & \multicolumn{3}{c}{ Growth in Plommet media } \\
\cline { 2 - 5 } Isolate & No carbon source & Erythritol & Ectoine & Rhamnose \\
\hline O. anthropi & $-(0.05)$ & $+(0.96)$ & $+(0.53)$ & $+(1.12)$ \\
B13-0095 & $-(0.03)$ & $+(1.03)$ & $+(0.64)$ & $+(0.98)$ \\
BO1 & $-(0.03)$ & $+(1.03)$ & $-(0.03)$ & $+(0.92)$ \\
BO2 & $-(0.03)$ & $+(1.16)$ & $-(0.03)$ & $+(1.07)$ \\
NF2653 & $-(0.03)$ & $+(0.66)$ & $-(0.03)$ & $-(0.03)$ \\
B. suis 1330 & $-(0.03)$ & $+(0.57)$ & $-(0.03)$ & $-(0.02)$ \\
\hline
\end{tabular}

Carbon source usage was determined by the ability of the indicated strains to grow in a chemically defined medium containing either ectoine or rhamnose as a sole carbon source. Erythritol was used as a positive control. $+=$ growth, $-=$ no growth. Values in parenthesis indicate the $O D_{600}$ after $24 \mathrm{~h}$ of culture (except for $\mathrm{O}$. anthropi for which growth was measured after $48 \mathrm{~h}$.

these genes are involved in ectoine uptake (through an $\mathrm{ABC}$ type transporter) or degradation (Figure 2A). The gene doeC (encoding an aspartate-semialdehyde dehydrogenase) is present in this cluster, but was not listed as a B13-0095 specific gene (Table 3) because it is conserved in several other Brucella spp. We found none of the genes required for ectoine/hydroxyectoine 


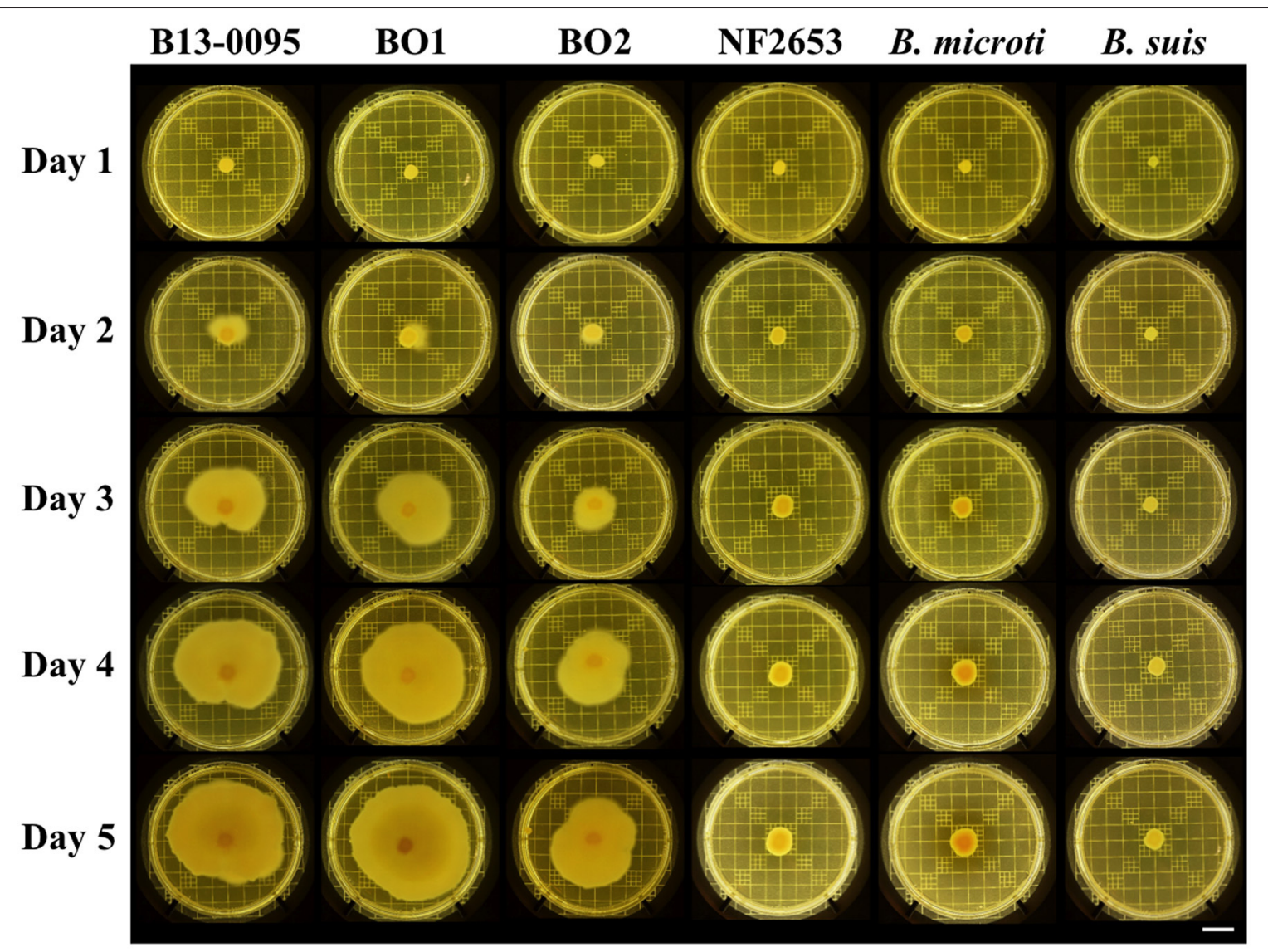

FIGURE 3 | Motility of Brucella strains. Motility of the indicated strains was visualized using on plate swimming assays. Shown are representative results of at least 3 independent repeats. Scale bar $=2 \mathrm{~cm}$.

(ectABCD) biosynthesis in the B13-0095 or O. anthropi genomes, suggesting that these bacteria cannot synthesize (hydroxy)ectoine de novo.

Ectoines (ectoin and hydroxyectoin) are water-soluble organic compounds produced by many halophilic bacteria. They protect these microorganisms from deleterious environmental conditions, such as osmotic, thermal, or hygrometric stress (Oren, 2008), however, neither B13-0095 nor O. anthropi showed thermo- or osmo-tolerance compared to $\mathrm{BO} 1$ and $\mathrm{BO} 2$, and exogenously provided ectoine did not restore the growth rate of B13-0095 under hyperosmotic stress (data not shown). Alternatively, ectoines can be used as carbon and nitrogen sources. To ascertain whether B13-0095 can do so, we tested its ability to grow in a chemically defined medium with ectoine as sole carbon source. While all Brucella species tested could use erythritol, only B13-0095 was able to use ectoine as a sole carbon source (Table 4), demonstrating a new catabolic pathway that is specific to B13-0095 among the Brucella genus.

\section{A Rhamnose Catabolism Pathway Unique to the BO Clade Brucella}

Among the genes conserved within the BO clade Brucella and O. anthropi, several genes, arranged as a cluster, are involved in L-Rhamnose (rhamnose) uptake and catabolism (Table S4). Rhamnose is a sugar commonly found in nature; it is a constituent of the cell wall of some plants and a potential carbon source for plant-associated bacteria (Eagon, 1961). Its catabolism also plays a significant role in some bacteria-plant interactions, e.g., nodulation of rhizobia (Oresnik et al., 1998).

The genes found in this locus, conserved in B13-0095, BO1, $\mathrm{BO} 2$, and $\mathrm{O}$. anthropi, were predicted to encode a set of rhamnose catabolic enzymes (RhaI, RhaD, and RhaK), an ABC-type rhamnose transport system (RhaS, RhaT, RhaP, and RhaQ) and a DeoR-type transcriptional regulator (RhaR) (Figure 2B). The gene encoding a rhamnose mutarotase (RhaU) was not identified through the PATRIC FigFam search, but a BLASTN search confirmed that this gene is indeed present in the genomes of $\mathrm{B} 13$ 0095, BO1, BO2, and O. anthropi (peg.238, WP_008511386.1, WP_009363826.1, and WP_012092962.1 respectively), but not in any other brucellaceae. In B13-0095 and the BO clade Brucella, as well as in O. anthropi, the genes rhaRSTPQUK are oriented divergently from rhaDI. In O. anthropi this genetic cluster is very distant from the genes encoding the flagella apparatus, which are arranged as 3 successive loci (Figure 2C and Table S3). In Brucella genomes however, the 3 flagella loci are separated and contain, between flagella loci II and III, a genomic fragment of 14 


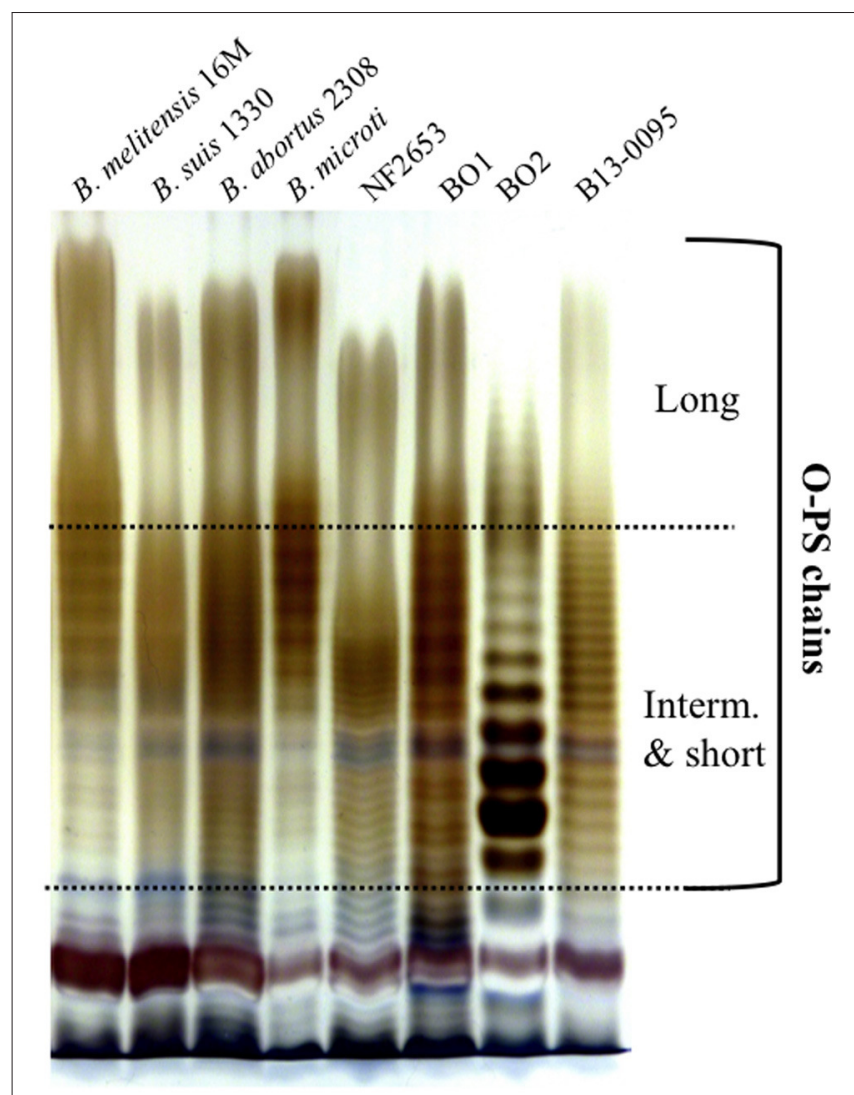

FIGURE 4 | Electrophoretic profiles of the LPS produced by different Brucella. Silver staining after SDS-PAGE of proteinase K-digested LPS preparations from the indicated Brucella strains. The bands corresponding to the long, intermediate or short sugar chains of O-PS are indicated.

$\mathrm{kb}$ including genes involved in several metabolic pathways (lipid or carbohydrates) or in resistance to cationic antimicrobials. In the BO clade of Brucella however, the rhamnose locus is located in the immediate vicinity of the flagella locus II.

Using chemically defined media, we found that B13-0095, $\mathrm{BO} 1, \mathrm{BO} 2$, and $O$. anthropi can use rhamnose as a carbon source (Table 4), highlighting a new catabolic pathway specific to this group of Brucella.

\section{The Brucella BO Clade Are Motile Bacteria}

Brucella are historically classified as non-motile, however Eisenberg et al. (2012) recently reported that Brucella isolates from African bullfrogs were motile. Using swimming motility assays on soft-agar plates, we found that B13-0095 is also highly motile, the bacterial layer covering a surface of up to $28 \mathrm{~cm}^{2}$ in 5 days (Figure 3). Interestingly, $\mathrm{BO} 1$ and $\mathrm{BO} 2$ are also highly motile, while NF2653 (closest relative to the BO clade), B. microti (a non-related, fast-growing strain) and B. suis (classical Brucella) are non-motile.

Although non-motile, the genomes of the classical species contain all the genes necessary to assemble an active flagellum, however several are pseudogenes (Letesson et al., 2002; Chain et al., 2005). Analysis of all flagella related genes previously predicted to be pseudogenes in either B. melitensis $16 \mathrm{M}, B$. suis 1330, or B. abortus 2308 (Chain et al., 2005) confirmed the absence of pseudogenes in B13-0095 or BO1 and BO2 (Table 5). However, the genes encoding the flagellar biosynthesis protein FlhA and the flagellar M-ring protein FliF are both pseudogenes in strain NF2653, which was not motile in our assays. This suggests that the BO clade Brucella, including B13-0095, may be able to assemble functional flagella, explaining their motility.

\section{The Pac-Man Frog Brucella Isolate Produces an Atypical LPS}

As reported for the African bullfrog isolates (Eisenberg et al., 2012), B13-0095 does not agglutinate with Brucella specific sera. This suggested that, as seen for $\mathrm{BO} 2$, this strain does not synthesize the typical Brucella perosamine based O-antigen (Wattam et al., 2012). We thus analyzed the LPS produced by B13-0095 by SDS-PAGE and silver staining and compared the pattern obtained to that of BO1, BO2, or NF2653, as well as smooth classical Brucella strains (Figure 4). The $\mathrm{O}$-antigen of B13-0095 displayed a continuous ladder-like pattern typical of a smooth LPS in which O-antigen is made of repeated oligosaccharide units. This pattern, with very close and regularly spaced narrow bands, looked similar to that observed with BO1 and NF2653. However, contrary to the other smooth LPS displaying a classical bimodal distribution (i.e., containing both long- and intermediate-chains of polysaccharides), the B13-0095 $\mathrm{O}$-antigen seems to be predominantly composed of short- and intermediate-chains of polysaccharide.

We then analyzed the genes involved in LPS biosynthesis in the B13-0095 genome, focusing first on the $w b k$ and $w b o$ regions that contain the genes involved in $\mathrm{O}$-polysaccharide synthesis in classical Brucella. We found that, like BO2, B13-0095 lacks the $w b o A$ and $w b o B$ genes (Table S4). The organization of the $w b k$ region is also similar to that in $\mathrm{BO} 2$, with 11 missing genes that are replaced by several others. Four of them $(\mathrm{rmlA}, \mathrm{rmlC}, \mathrm{rmlB}$, and $r m l D$ ) are used in some bacteria for making a rhamnosebased O-antigen (Figure 5 and Table S4). The others encode a glycosyltransferase, a hypothetical protein and two genes $(r f b B$ and $r f b D$ ) predicted to be involved in $\mathrm{O}$-antigen export but that differ from those with the same function in the classical Brucella ( $w z m$ and $w z t$ ). It should be noted that the genes BIBO2_1979 to $\mathrm{BIBO} 2 \_1982$ in the $w b k$ region of $\mathrm{BO} 2$ were not described by Wattam et al. (2012), probably because they are contained in a small contig of $2.14 \mathrm{~kb}$ (contig ADFA01000111). Another feature specific to B13-0095 is the lack of three genes $(x y l G$, $x y l H$, and $x y l F$ ) that are found in the immediate vicinity of the $w b k$ region in classical Brucella and predicted to encode a xylose import system. The genes encoding the enzymes involved in the modification of sugar precursors $\left(p g m, m a n B_{\text {core }}, m a n C_{\text {core }}\right.$, $k d s A$, and $k d s B$ ) or incorporation of sugars (wadA, wadB, and wadC) into Brucella LPS are present in B13-0095 genome. All the genes involved in lipid A biosynthesis are also present in B13-0095 genome, however region 2 contains an additional fragment of $6.5 \mathrm{~kb}$ encoding hypothetical or phage proteins. Finally, PATRIC identified several other genes putatively related to LPS in B13-0095 genome, all of them being present in all the 
TABLE 5 | Analysis of the flagella related genes in the BO clade Brucella.

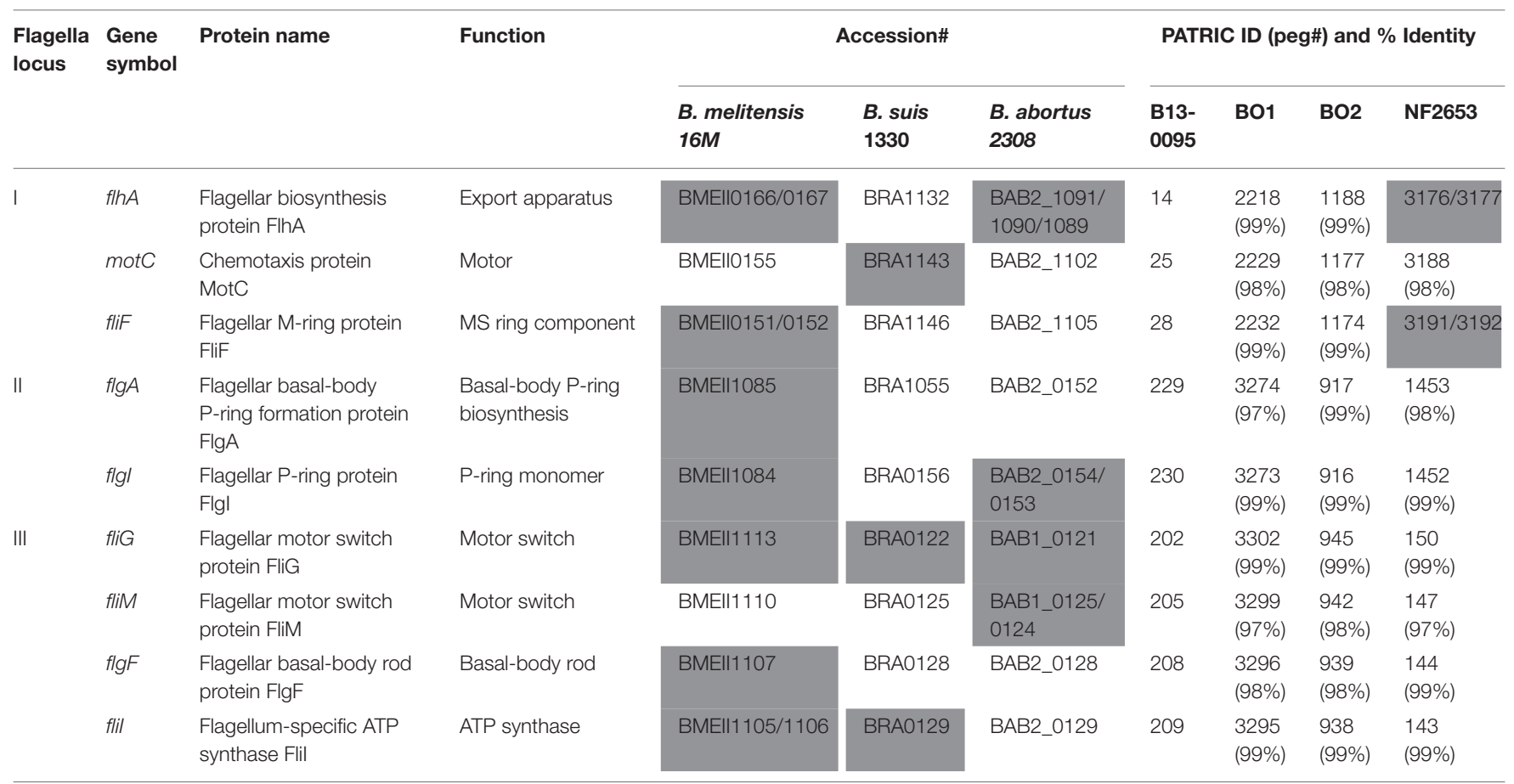

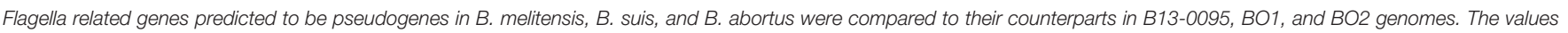
in brackets are the \% of identity at the protein level when compared with the corresponding gene in B13-0095 genome. Pseudogenes are shown in gray boxes.

Brucella analyzed in this study. Among them, $l p t A, l p t B$, and $l p t C$ are predicted to encode proteins involved in LPS export to the outer membrane. The Lpt system is encoded by 7 genes in E. coli (Sperandeo et al., 2009); search for orthologs identified the genes encoding the 4 remaining proteins, which are conserved across Brucella.

\section{The Pac-Man Frog Brucella Isolate Has a Fast Replication Rate within Eukaryotic Cells}

Brucella are facultative intracellular pathogens. Their ability to replicate inside eukaryotic cells, the major feature for their pathogenic potential, is highly dependent on the structure of their LPS (Porte et al., 2003; Mancilla, 2016). We thus measured the intracellular replication rate of B13-0095 in human epithelial cells (HeLa) and murine macrophages (J774), compared to B. suis strain 1330, with replication and intracellular trafficking profiles that are well characterized. We found that B13-0095 grows much faster within both HeLa and J774 cells (Figure 6). At 24 h postinfection (hpi), the intracellular bacterial numbers are 100-1000 times higher than observed with B. suis in HeLa or J774 cells, respectively, and reminiscent of that observed with BO1 (Jiménez de Bagüés et al., 2014). The $B$. suis $\Delta$ virB8 mutant provides a control showing that both cell lines are able to control infection by attenuated strains. Thus, the elevated rate of replication of the frog isolate cannot be attributed to a reduced fitness of these cells.

Low magnification observation of cells infected with fluorescent bacteria at 24 hpi suggested that, with B13-0095, there were more cells infected and that infected cells contained more bacteria (data not shown). This was confirmed using a flow cytometry analysis in which the level of infection is given by the $\%$ of fluorescent cells and the rate of intracellular replication is given by the median fluorescence intensity (MFI) of infected cells. We observed that B13-0095 infects more HeLa cells than B. suis and replicates much faster within infected cells (Figure 7). High-resolution observations of infected cells revealed many more bacteria at $24 \mathrm{hpi}$ with the frog isolate than with $B$. suis (Figure 8). At $24 \mathrm{~h} \mathrm{pi,} \mathrm{the} \mathrm{cytoplasm} \mathrm{of} \mathrm{cells} \mathrm{infected} \mathrm{with}$ B13-0095 were already fully packed with bacteria, while this is usually observed after 48 hpi with $B$. suis. Identical observations were made with $\mathrm{BO} 1$, but not with $\mathrm{BO} 2$ (K. Garcia-Mendez and A. Keriel, unpublished data). B13-0095 is more prolific with regard to infection and replication within eukaryotic cells than classical Brucella.

\section{DISCUSSION}

\section{Potential Sources of Infection in Frogs}

Over the first years of the 21st century the Brucella genus has expanded from 6 to 11 known species, all of which were associated with mammals. In 2012, the natural hosts range expanded to amphibians after the reports of atypical strains from frogs. The first cases described in Germany (Big-eyed tree frog and African bullfrogs, Eisenberg et al., 2012; Fischer et al., 2012) were in animals imported from Africa, while the White's tree frog in the UK belongs to a species originally native to Australia (Whatmore et al., 2015). B13-0095 is the first 


\section{Classical Brucella and BO1}
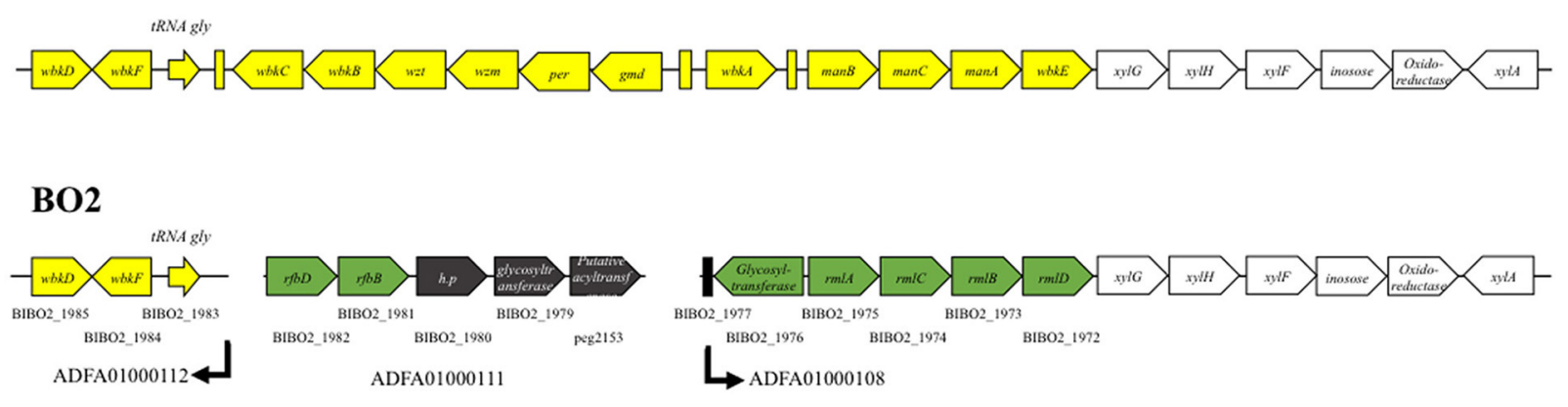

\section{B13-0095}
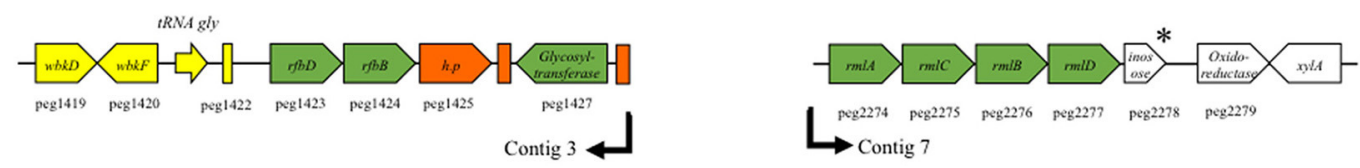

FIGURE 5 | Schematic representation of the wbk region organization in Brucella. The genes described in classical Brucella and B. inopinata BO1 within or flanking the wbk region are shown in yellow or white, respectively. The genetic features present in both the Pac-Man frog isolate B13-0095 and the B. inopinata-like strain $\mathrm{BO} 2$ genomes are shown in green. Features in black are only present in BO2 and features in orange only in B13-0095. The annotation of the genes is indicated, as well as their ID number in PATRIC (peg\#) or RefSeq (BIBO2\#). The arrows indicate the end/beginning of the contigs in the draft genome sequences of B13-0095 and BO2. * indicate predicted pseudogenes. Rectangles represent predicted mobile elements.

Brucella strain isolated from an amphibian indigenous to the Americas.

In the Big-eyed and White's tree frogs, bacteria were isolated from superficial lesions that did not affect the animal's overall health, whereas the African bullfrogs were found dead or moribund, similar to the Pac-Man frog described in this study. All these frogs were captive, being either pets (Big-eyed tree and Pac-Man frogs) or part of tropical animal collections (White's tree frog and African bullfrogs), and had limited contacts with exterior environment. The actual sources of the infections, or whether the animals were infected in the wild or during their time in captivity, are unknown. Possibly the mice used to feed the big frog species (Pac-Man and African bullfrogs) could have been the source of a foodborne, fatal infection. In this respect, the White's and Big-eyed tree frogs, that had only superficial infections, are not fed with mice but with insects or worms. Alternatively, the bacteria could be a contaminant in the substrates added in the terrarium of the animals, such as coco husk, bark chippings, or plants, or in live moss, which is often used to maintain housing humidity. Some of the specific genetic features of the Pac-Man frog isolate B13-0095 are shared with bacteria of the rhizosphere (see below), suggesting that these bacteria could be associated with plants roots or found in the soil.

\section{Early-Diverging Brucella As an Intermediate Stage between a Soil Associated Ancestor and Host Adapted Species}

Analysis of Brucella genome sequences has begun to unearth the pathway of evolution from an environmental organism to a stealth pathogen. This involved acquisition of genetic material encoding virulence factors combined with genome reduction (Wattam et al., 2014b). Phylogenetically, Brucella divide into two clades, the "classical" Brucella and an early dividing branch of atypical strains. The kSNP analysis (Figure 1) shows that B13-0095 belongs to the group of early-diverging brucellae with traits that depart significantly from those of the classical Brucella spp. This group includes the two strains that form the " $\mathrm{BO}$ clade" (B. inopinata $\mathrm{BO} 1$ and strain $\mathrm{BO} 2$ ), a group of strains from Australian rodent strains and the previously reported frog isolates. MLST analysis has highlighted the genetic heterogeneity among the frog isolates (Eisenberg et al., 2012; Whatmore et al., 2015), suggesting that B13-0095 may also form a separate branch in this group. Phylogenetic placement of B13-0095 compared to the other frog isolates using WGS data will help understanding the evolutionary and phylogenetic relationships among the amphibian isolates.

Several features in B13-0095 genome suggest that it represents another stage in the evolution of Brucella from a soil associated ancestor to the host adapted "classical" species. Two regions endow metabolic capacities associated with soil bacteria that the "classical" Brucella have lost. An ectoine utilization gene cluster, conserved in $O$. antropi and some environmental halophilic bacteria, allows B13-0095 to use ectoine as a carbon source. Another genetic region (already noted in Wattam et al., 2012), which is conserved within the BO clade Brucella and O. anthropi, contains genes encoding rhamnose uptake and utilization systems. The genes are organized as those on a plasmid from $R$. leguminosarum, where this locus has been extensively studied (Oresnik et al., 1998). We found that B13-0095, BO1, and $\mathrm{BO} 2$ (but no other Brucella) have the ability to use 


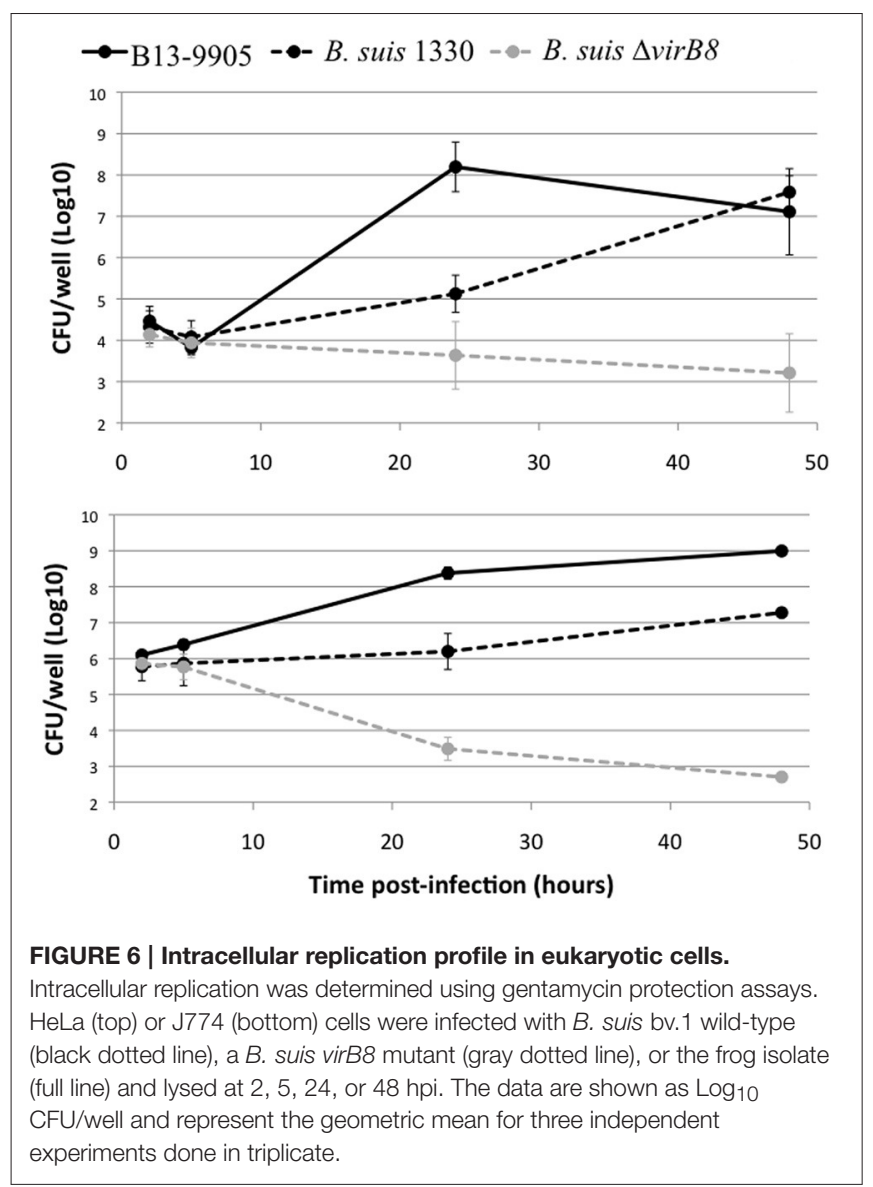

rhamnose as a carbon source. This may be linked to the closer proximity of the BO clade Brucella to environmental ancestors. Rhamnose is commonly found in plants as a part of complex pectin polysaccharides. These compounds can be degraded by saprophytic or plant-pathogenic bacteria using a set of extracellular enzymes (Rodionova et al., 2013). The resulting plant-derived rhamnose can then be used as a carbon source by microorganisms in the rhizosphere. We did not find any genes involved in the degradation of plants compounds in B130095 genome, suggesting that, if these bacteria can reside in the rhizosphere, they would only be able to use rhamnose made available by other organisms in this symbiotic community.

\section{Atypical O-PS in Brucella: Role in Virulence?}

Our bioinformatics analysis has highlighted novel features concerning the genetics of LPS synthesis in B13-0095 and has also given new information on the LPS export applicable to all Brucella. With the exception of the two naturally rough species (B. canis and B. ovis) Brucella were traditionally reported to have a perosamine based O-antigen. In the classical Brucella, mannose is used as a sugar precursor for the synthesis of $\mathrm{N}$ formylperosamine, which is then transferred to a bactoprenol molecule by several glycosyltransferases (WbkA, WbkE, WboA, and WboB) (Figure 9). The O-PS is then exported from the
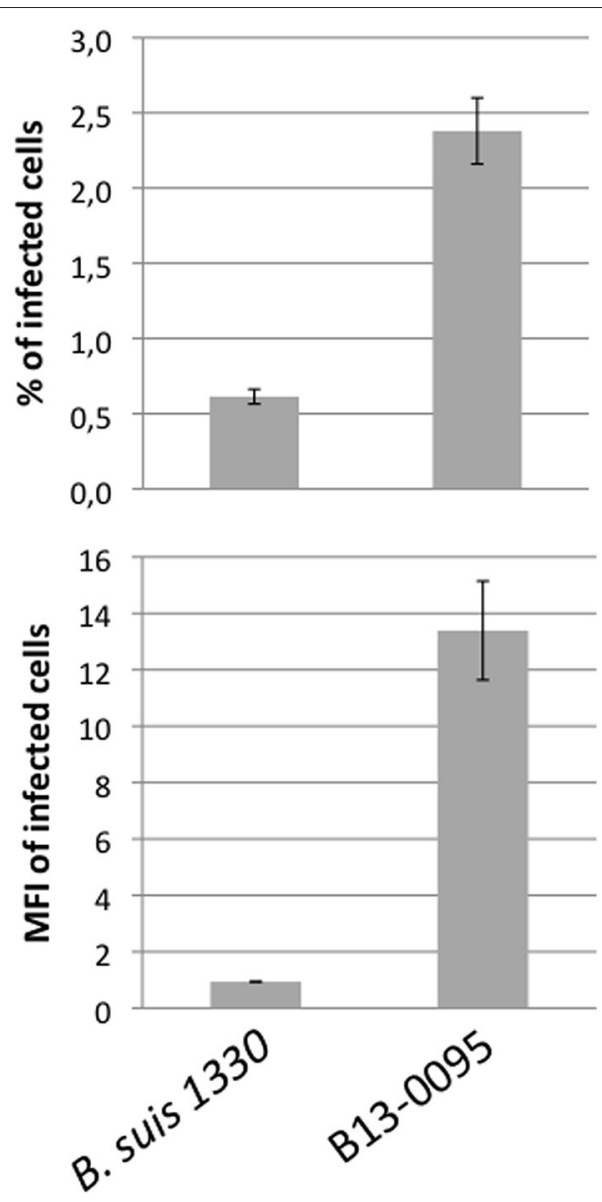

FIGURE 7 | Quantification of infection rate by flow cytometry. HeLa cells were infected by mCherry-expressing bacteria, detached, and fixed $24 \mathrm{hpi}$ and analyzed by flow cytometry. The level of infection is given by the $\%$ of infected (i.e., mCherry ${ }^{+}$) cells (top). The fluorescence intensity of infected cells was quantified by the MFI (Median Fluorescence Intensity) values (bottom), which is indicative of the intracellular replication rate of the bacteria. The data are mean values ( \pm SEM) calculated on 3 replicates.

cytoplasm to the outer leaflet of the inner membrane (periplasm) using a system encoded by $w z m$ and wzt. We found that, as with $\mathrm{BO} 2, \mathrm{~B} 13-0095$ lacks many of the genes of the $w b k$ region involved in the synthesis of this perosamine-based O-PS and has instead genes that several plant-associated bacteria use for making a rhamnose-based O-PS (Jofré et al., 2004; Broughton et al., 2006; Balsanelli et al., 2010; Clifford et al., 2013). B130095 and $\mathrm{BO} 2$ possess all the enzymes required to synthesize dTDP-rhamnose from glucose. This modified sugar may then be transferred to bactoprenol by the putative glycosyltransferases encoded by the genes BIBO2-1976/peg.1427, BIBO2-1980, or by other glycosyltransferases encoded elsewhere in their genomes. The O-PS could then be translocated to the periplasm using RfbB and $\mathrm{RfbD}$, both predicted to be involved in $\mathrm{O}$-antigen export.

LPS is considered as a major virulence factor in Brucella (Porte et al., 2003). The structure of the core plays a role in the ability to avoid recognition by the innate immune response (Fontana et al., 2016) and a complete, perosamine based O-PS 


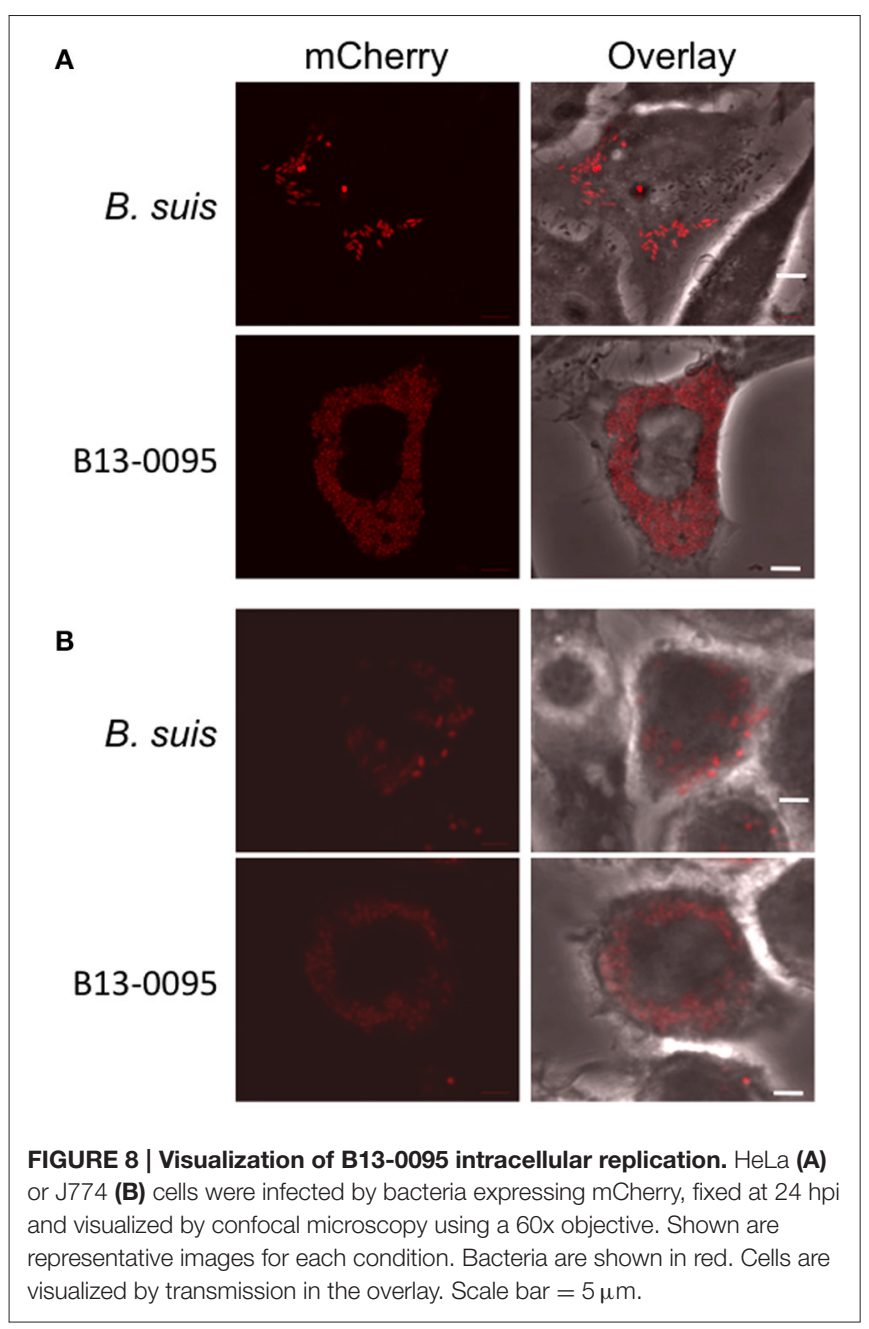

has been shown to be essential for host-pathogens interactions for the smooth classical Brucella, being involved in an interaction with lipid rafts at the cell surface that allow bacteria to enter and traffic through a pathway targeting them to an appropriate intracellular replicative niche (Porte et al., 2003). The low intracellular replication rate of Brucella strain BO2 (B. Saadeh and D. O'Callaghan, unpublished data), which has a rhamnose based $\mathrm{O}$-antigen, led to the suggestion that the acquisition of a perosamine based LPS was one of the key steps in the evolution of Brucella virulence (Wattam et al., 2012). However, the fast intracellular multiplication of B13-0095 shows that other factors are also important. Future work will be required to characterize biochemically the O-antigen produced by B13-0095 and determine its role in virulence.

After linking the O-PS to core-lipid A in the periplasm, the assembled LPS molecules must be shuttled to the outer membrane. In several Gram-negative bacteria, the lipopolysaccharide transport (Lpt) machinery is responsible for the export of LPS from the periplasmic surface of the inner membrane, across the periplasm, to the outer leaflet of the outer membrane (Sperandeo et al., 2009). The seven proteins in the Lpt system (LptA to LptG) constitute a large complex spanning the inner to the outer membrane and operating as a concerted machine. The identification of all the genes encoding the Lpt export system in Brucella adds another step in our comprehension of LPS synthesis in this genus.

\section{The BO-Clade Brucella Are Motile}

Classically, Brucella are classified as non-motile bacteria. One of the major finding in our study is the demonstration that the atypical BO clade Brucella, including B13-0095, BO1, and BO2, are highly motile. Motility among Brucella isolates from African bullfrogs was reported (Eisenberg et al., 2012) however, as no data was presented, it is not possible to compare motility efficiencies among these strains. Importantly, it should be noted that the tests performed in agar tubes upon initial isolation of B13-0095 in Texas did not allow detection of the motility of this organism, suggesting that these tests, commonly used for biotyping, may not be optimal for some bacterial species. In this respect, the initial reports on $\mathrm{BO} 1$ and $\mathrm{BO} 2$, which probably also used in tube assays, describe both strains as non-motile (De et al., 2008; Tiller et al., 2010a).

During evolution, the classical Brucella have accumulated mutations that have led to the formation of several pseudogenes among flagella related genes (Letesson et al., 2002; Chain et al., 2005; Tsolis et al., 2009). Despite these mutations, under specific growth conditions, these strains can make a sheathed flagellum that does not confer motility, but is required for virulence (Fretin et al., 2005; Petersen et al., 2011). Analysis of the flagella genes in B13-0095, BO1, and BO2 showed that all the genes were fully functional, probably explaining the motility of these three strains. The second group of atypical strains, isolated from Australian rodents and represented by NF2653, are non-motile and also have flagella pseudogenes. This gives us a starting point for the process of flagella degradation. Motility in the BO clade Brucella is probably a residual property from environmental ancestors that could provide a strong advantage by allowing it to move to a favorable niche. Understanding the role of this motility in the virulence of these strains and their intracellular lifestyle will require further studies. Motility could provide an advantage at different stages of their pathogenic cycle: reaching the host, invading and colonizing the host cells, and dispersing to new hosts. At the cellular level, motility could help the bacteria reach a permissive cell or entry site, as it has been shown for Salmonella that "near surface-swimming" motility enhances bacterial uptake (Misselwitz et al., 2012). A role for motility in reaching the host cell surface would be consistent with an elevated rate of infection observed for B13-0095 (this study), BO1 and BO2 (B. Sadeeh and D. O'Callaghan, unpublished data). It would also be of interest to determine whether the FliC flagellin encoded by the BO clade Brucella lacks the TLR5 antagonist property demonstrated in $B$. melitensis (Terwagne et al., 2013).

\section{Risks of Misidentification of Atypical Brucella Isolates}

Accurate identification of pathogens is essential for establishing dependable diagnosis, choosing a treatment, and understanding the source of infection. This study highlights the risk that atypical Brucella spp. isolated from amphibians may be misidentified as 


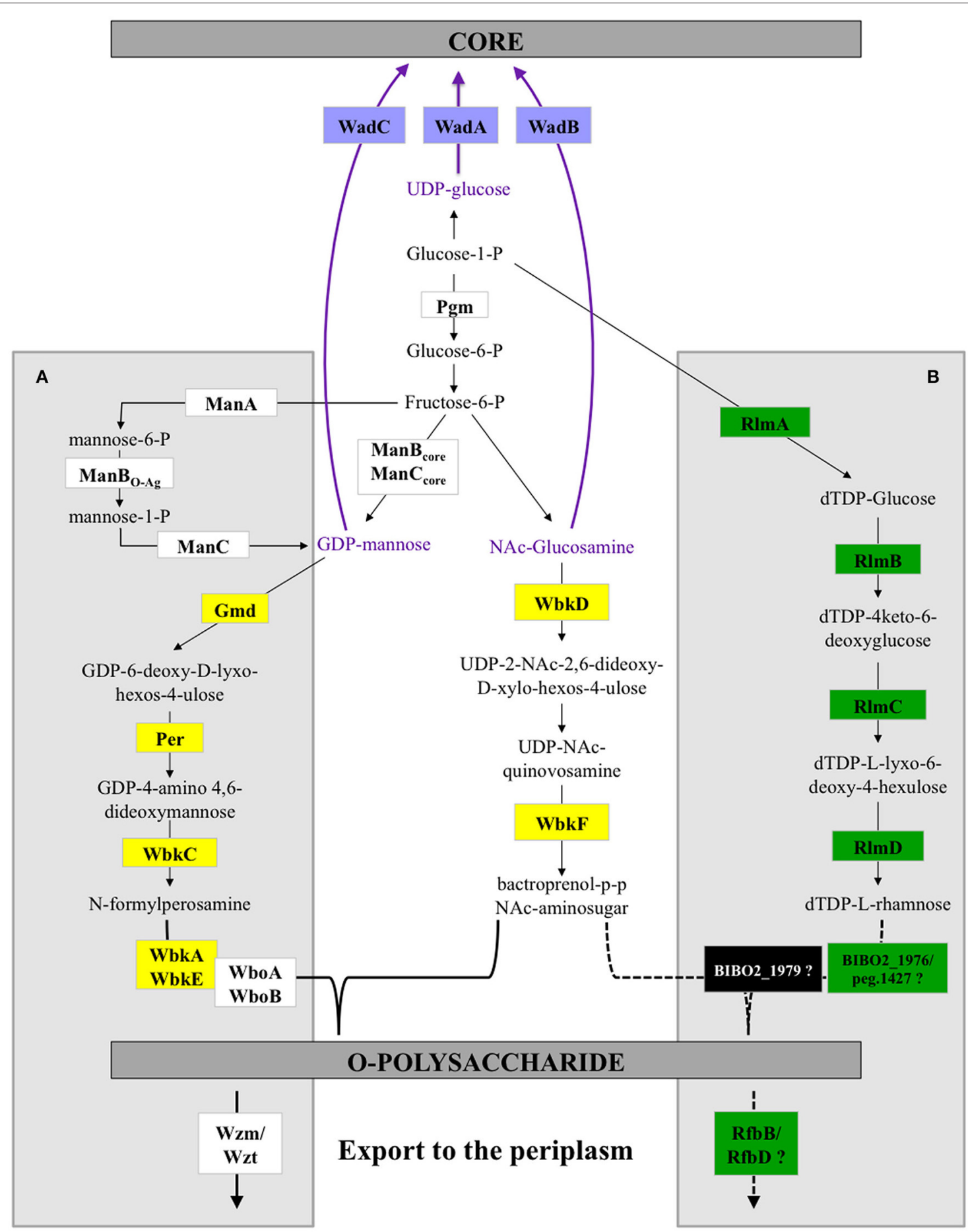

FIGURE 9 | LPS biosynthesis pathways in Brucella. Schematic representation of the O-polysaccharide biosynthesis pathways in Brucella. The A box highlights the reactions previously described for classical Brucella. The B box shows the predicted alternative pathways in the Pac-Man frog isolate B13-0095 and strain BO2. The reactions outside the boxes are common. Dotted lines show speculated reactions. The color code used to highlight some enzymes in this scheme is the same as in Figure 5 and Table S4.

O. anthropi, an opportunistic pathogen in humans, particularly in immunocompromised patients, which has been associated with wound infections, abscesses or septicemia (Kettaneh et al., 2003;
Ozdemir et al., 2006; Vaidya et al., 2006; Hagiya et al., 2013). O. antropi is widely distributed in the environment (water, plants, soil) and can contaminate indwelling medical devices. Infections 
with $O$. anthropi have also been reported in free-ranging amphibians (cane toads) in Australia (Brown et al., 2007; Shilton et al., 2008). Despite being bona fide Brucella, the frog isolates were not identified as such. First, the rapid growth rate excluded the identification as Brucella as did the lack of agglutination with specific antisera. In many clinical laboratories, the initial identification is made using the commercially available tests such as API 20NE. However, we and others found that API 20NE tests misidentify fast growing atypical Brucella spp. such as $O$. anthropi with a very high confidence level (Scholz et al., 2010; Tiller et al., 2010a; Fischer et al., 2012). Thus, in all human and animal cases in which identification of the pathogen as $O$. anthropi was solely based on this test (including the cane toads cited above), involvement of atypical Brucella spp. cannot be excluded. Matrix assisted laser desorption/ionization-time of flight (MALDI-TOF) mass spectrometry (MS) is rapidly becoming the method of choice for bacterial identification in clinical laboratories. It allows a fast identification of bacteria and yeast, but its accuracy largely depends on the coverage of the database. With regard to Brucella, accurate identification by MALDI-TOF MS is currently limited because this genus is either not represented in the databases of the two main manufacturers, or only in a bioterrorism database with restricted availability (Cunningham and Patela, 2013). Using a safe inactivation protocol developed recently (Mesureur et al., 2016), we constructed an exhaustive MALDI-TOF MS Brucella database that can identify all Brucella spp., in most cases at the species level (J. Mesureur and A. Keriel, unpublished data). This database allowed us to clearly discriminate the frog isolates B130095 from $O$. anthropi and identified it as B. inopinata (this data will be presented in a separate publication). Another possibility would be to exploit the absence of some genes (shown in red in Figure 2 and Table S3) from Ochrobactrum spp. genomes to discriminate between atypical Brucella and O. anthropi using PCR.

Isolation of Brucella spp. from frogs from Africa, Australia and America suggests that they may be widespread and highlight

\section{REFERENCES}

Alton, G. G., Jones, L. M., Angus, R. D., and Verger, J. M. (1988). Techniques for the Brucellosis Laboratory. Paris: Institut National de la Recherche Agronomique (INRA).

Audic, S., Lescot, M., Claverie, J.-M., and Scholz, H. C. (2009). Brucella microti: the genome sequence of an emerging pathogen. BMC Genomics 10:352. doi: 10.1186/1471-2164-10-352

Balsanelli, E., Serrato, R. V., de Baura, V. A., Sassaki, G., Yates, M. G., Rigo, L. $\mathrm{U}$., et al. (2010). Herbaspirillum seropedicae $\mathrm{rfbB}$ and $\mathrm{rfbC}$ genes are required for maize colonization. Environ. Microbiol. 12, 2233-2244. doi: 10.1111/j.14622920.2010.02187

Barbier, T., Collard, F., Zúñiga-Ripa, A., Moriyón, I., Godard, T., Becker, J., et al. (2014). Erythritol feeds the pentose phosphate pathway via three new isomerases leading to D-erythrose-4-phosphate in Brucella. Proc. Natl. Acad. Sci. U.S.A. 111, 17815-17820. doi: 10.1073/pnas.1414622111

Broughton, W. J., Hanin, M., Relic, B., Kopciñska, J., Golinowski, W., Simsek, S., et al. (2006). Flavonoid-inducible modifications to rhamnan $\mathrm{O}$ antigens are necessary for Rhizobium sp. strain NGR234-legume symbioses. J. Bacteriol. 188, 3654-3663. doi: 10.1128/JB.188.10.3654-3663.2006

Brown, G. P., Shilton, C., Phillips, B. L., and Shine, R. (2007). Invasion, stress, and spinal arthritis in cane toads. Proc. Natl. Acad. Sci. U.S.A. 104, 17698-17700. doi: 10.1073/pnas.0705057104 a need for a broader assessment of the presence of Brucella in amphibians worldwide. Thus, not only better identification tools are required, but prevention measures should also be taken. Whilst the zoonotic potential of this group is not known yet, their close proximity with strains associated with human disease suggests that they might present a risk to the animals' keepers and thus, unnecessary contact with potentially infected amphibians should be avoided.

\section{AUTHORS CONTRIBUTIONS}

PS, CQ, DO, and AK designed the study; PS, SL, TS, JE, and AK acquired the data; PS, CQ, SL, TS, DO, and AK analyzed and interpreted the data; PS, CQ, SL, JE, TF, SR, DO, and AK participated in drafting the article or revising it critically.

\section{FUNDING}

This work was supported by the Institut National de la Santé et de la Recherche Médicale (INSERM), Université de Montpellier and the Institut de Veille Sanitaire (InVS). PFSL is recipient of a post-doctoral research grant from the Fondation Méditerranée Infection (France).

\section{ACKNOWLEDGMENTS}

Strains $\mathrm{BO} 1, \mathrm{BO} 2$, and NF2653 were provided by Barun De (CDC, Atlanta, GO, USA). B. microti CCN4915 was kindly provided by Alessandra Occhialini (CPBS, Montpellier, France).

\section{SUPPLEMENTARY MATERIAL}

The Supplementary Material for this article can be found online at: http://journal.frontiersin.org/article/10.3389/fcimb. 2016.00116

Celli, J. (2006). Surviving inside a macrophage: the many ways of Brucella. Res. Microbiol. 157, 93-98. doi: 10.1016/j.resmic.2005.10.002

Celli, J. (2015). The changing nature of the Brucella-containing vacuole. Cell. Microbiol. 17, 951-958. doi: 10.1111/cmi.12452

Chain, P. S. G., Comerci, D. J., Tolmasky, M. E., Larimer, F. W., Malfatti, S. A., Vergez, L. M., et al. (2005). Whole-genome analyses of speciation events in pathogenic Brucellae. Infect. Immun. 73, 8353-8361. doi: 10.1128/IAI.73.12.8353-8361.2005

Clifford, J. C., Rapicavoli, J. N., and Roper, M. C. (2013). A rhamnose-rich Oantigen mediates adhesion, virulence, and host colonization for the xylemlimited phytopathogen Xylella fastidiosa. Mol. Plant Microbe Interact. 26, 676-685. doi: 10.1094/MPMI-12-12-0283-R

Cunningham, S. A., and Patela, R. (2013). Importance of using bruker's securityrelevant library for biotyper identification of Burkholderia pseudomallei, Brucella species, and Francisella tularensis. J. Clin. Microbiol. 51, 1639-1640. doi: 10.1128/JCM.00267-13

De, B. K., Stauffer, L., Koylass, M. S., Sharp, S. E., Gee, J. E., Helsel, L. O., et al. (2008). Novel Brucella strain (BO1) associated with a prosthetic breast implant infection. J. Clin. Microbiol. 46, 43-49. doi: 10.1128/JCM.01494-07

De Figueiredo, P., Ficht, T. A., Rice-Ficht, A., Rossetti, C. A., and Adams, L. G. (2015). Pathogenesis and immunobiology of brucellosis: review of Brucellahost interactions. Am. J. Pathol. 185, 1505-1517. doi: 10.1016/j.ajpath.2015. 03.003 
Eagon, R. G. (1961). Bacterial dissimilation of L-fucose and L-rhamnose. J. Bacteriol. 82, 548-550.

Eisenberg, T., Hamann, H.-P., Kaim, U., Schlez, K., Seeger, H., Schauerte, N., et al. (2012). Isolation of potentially novel Brucella spp. from frogs. Appl. Environ. Microbiol. 78, 3753-3755. doi: 10.1128/AEM.07509-11

Fischer, D., Lorenz, N., Heuser, W., Kämpfer, P., Scholz, H. C., and Lierz, M. (2012). Abscesses associated with a Brucella inopinata-like bacterium in a bigeyed tree frog (Leptopelis vermiculatus). J. Zoo Wildl. Med. 43, 625-628. doi: 10.1638/2011-0005R2.1

Fontana, C., Conde-Alvarez, R., Ståhle, J., Holst, O., Iriarte, M., Zhao, Y., et al. (2016). Structural studies of lipopolysaccharide-defective mutants from Brucella melitensis identify a core oligosaccharide critical in virulence. J. Biol. Chem. 291, 7727-7741. doi: 10.1074/jbc.M115.701540

Foster, G., Osterman, B. S., Godfroid, J., Jacques, I., and Cloeckert, A. (2007). Brucella ceti sp. nov. and Brucella pinnipedialis sp. nov. for Brucella strains with cetaceans and seals as their preferred hosts. Int. J. Syst. Evol. Microbiol. 57:2688-2693. doi: 10.1099/ijs.0.65269-0

Fretin, D., Fauconnier, A., Köhler, S., Halling, S., Léonard, S., Nijskens, C., et al. (2005). The sheathed flagellum of Brucella melitensis is involved in persistence in a murine model of infection. Cell. Microbiol. 7:687-698. doi: 10.1111/j.14625822.2005.00502.x

Gardner, S. N., and Hall, B. G. (2013). When whole-genome alignments just won't work: kSNP v2 software for alignment-free SNP discovery and phylogenetics of hundreds of microbial genomes. PLoS One 8:e81760. doi: 10.1371/journal.pone. 0081760

Ha, D.-G., Kuchma, S. L., and O’Toole, G. A. (2014). "Plate-based assay for swimming motility in Pseudomonas aeruginosa," in Pseudomonas Methods and Protocols, eds A. Filloux and J.-L. Ramos (New York, FL: Springer Science), 59-65.

Hagiya, H., Ohnishi, K., Maki, M., Watanabe, N., and Murasec, T. (2013). Clinical characteristics of Ochrobactrum anthropi bacteremia. J. Clin. Microbiol. 51, 1330-1333. doi: 10.1128/JCM.03238-12

Jiménez de Bagüés, M. P., Iturralde, M., Arias, M. A., Pardo, J., Cloeckaert, A., and Zygmunt, M. S. (2014). The new strains Brucella inopinata BO1 and Brucella species 83-210 behave biologically like classic infectious Brucella species and cause death in murine models of infection. J. Infect. Dis. 210, 467-472. doi: 10.1093/infdis/jiu102

Jofré, E., Lagares, A., and Mori, G. (2004). Disruption of dTDP-rhamnose biosynthesis modifies lipopolysaccharide core, exopolysaccharide production, and root colonization in Azospirillum brasilense. FEMS Microbiol. Lett. 231, 267-275. doi: 10.1016/S0378-1097(04)00003-5

Keriel, A., Botella, E., Estrach, S., Bragagnolo, G., Vergunst, A. C., Feral, C. C., et al. (2015). Brucella intracellular life relies on the transmembrane protein CD98 heavy chain. J. Infect. Dis. 211, 1769-1778. doi: 10.1093/infdis/jiu673

Kettaneh, A., Weill, F. X., Poilane, I., Fain, O., Thomas, M., Herrmann, J. L., et al. (2003). Septic shock caused by Ochrobactrum anthropi in an otherwise healthy host. J. Clin. Microbiol. 41, 1339-1341. doi: 10.1128/JCM.41.3.1339-1341.2003

Lacerda, T. L., Salcedo, S. P., and Gorvel, J.-P. (2013). Brucella T4SS: the VIP pass inside host cells. Curr. Opin. Microbiol. 16, 45-51. doi: 10.1016/j.mib.2012.11.005

Lapaque, N., Moriyon, I., Moreno, E., and Gorvel, J. P. (2005). Brucella lipopolysaccharide acts as a virulence factor. Curr. Opin. Microbiol. 8, 60-66. doi: 10.1016/j.mib.2004.12.003

Letesson, J.-J., Lestrate, P., Delrue, R.-M., Danese, I., Bellefontaine, F., Fretin, D., et al. (2002). Fun stories about Brucella: the "furtive nasty bug." Vet. Microbiol. 90, 317-328. doi: 10.1016/S0378-1135(02)00208-0

Mancilla, M. (2016). Smooth to rough dissociation in Brucella: the missing link to virulence. Front. Cell. Infect. Microbiol. 5:98.doi: 10.3389/fcimb.2015.00098

Mesureur, J., Ranaldi, S., Monnin, V., Girard, V., Arend, S., Welker, M., et al. (2016). A simple and safe protocol for preparing Brucella samples for matrixassisted laser desorption ionization-time of flight mass spectrometry analysis. J. Clin. Microbiol. 54, 449-452. doi: 10.1128/JCM.02730-15

Misselwitz, B., Barrett, N., Kreibich, S., Vonaesch, P., Andritschke, D., Rout, S., et al. (2012). Near surface swimming of Salmonella Typhimurium explains target-site selection and cooperative invasion. PLoS Pathog. 8:e1002810. doi: 10.1371/journal.ppat.1002810

Moreno, E. (2014). Retrospective and prospective perspectives on zoonotic brucellosis. Front. Microbiol. 5:213. doi: 10.3389/fmicb.2014.00213
Oren, A. (2008). Microbial life at high salt concentrations: phylogenetic and metabolic diversity. Saline Syst. 4:2. doi: 10.1186/1746-1448-4-2

Oresnik, I. J., Pacarynuk, L. A., O’Brien, S. A. P., Yost, C. K., and Hynes, M. F. (1998). Plasmid-encoded catabolic genes in Rhizobium leguminosarum bv. trifolii: evidence for a plant-inducible rhamnose locus iinvolved in competition for nodulation. Mol. Plant Microbe Interact. 11, 1175-1185.

Ozdemir, D., Soypacaci, Z., Sahin, I., Bicik, Z., and Sencan, I. (2006). Ochrobactrum anthropi endocarditis and septic shock in a patient with no prosthetic valve or rheumatic heart disease: case report and review of the literature. Jpn. J. Infect. Dis. 59, 264-265.

Paschos, A., Patey, G., Sivanesan, D., Gao, C., Bayliss, R., Waksman, G., et al. (2006). Dimerization and interactions of Brucella suis VirB8 with VirB4 and VirB10 are required for its biological activity. Proc Natl Acad Sci USA 103, 7252-7257. doi: 10.1073/pnas.0600862103

Petersen, E., Chaudhuri, P., Gourley, C., Harms, J., and Splitter, G. (2011). Brucella melitensis Cyclic di-GMP Phosphodiesterase BpdA Controls Expression of Flagellar Genes. J. Bacteriol. 193, 5683-5691. doi: 10.1128/JB.00428-11

Porte, F., Naroeni, A., Ouahrani-Bettache, S., and Liautard, J.-P. (2003). Role of the Brucella suis lipopolysaccharide $\mathrm{O}$ antigen in phagosomal genesis and in inhibition of phagosome-lysosome fusion in murine macrophages. Infect. Immun. 71, 1481-1490. doi: 10.1128/IAI.71.3.1481-1490.2003

Quance, C., Robbe-Austerman, S., Stuber, T., Brignole, T., DeBess, E. E., Boyd, L., et al. (2016). Identification of source of Brucella suis infection in human by using whole-genome sequencing, United States and Tonga. Emerging Infect. Dis. 22, 79-82. doi: 10.3201/eid2201

Rodionova, I. A., Li, X., Thiel, V., Stolyar, S., Stanton, K., Fredrickson, J. K., et al. (2013). Comparative genomics and functional analysis of rhamnose catabolic pathways and regulons in bacteria. Front. Microbiol. 4:407. doi: 10.3389/fmicb.2013.00407

Scholz, H. C., Al Dahouk, S., Hammerl, J. A., Zygmunt, M. S., Cloeckaert, A., Koylass, M., et al. (2016). Brucella vulpis sp. nov., isolated from mandibular lymph nodes of red foxes (Vulpes vulpes). Int. J. Syst. Evol. Microbiol. 66, 2090-2098. doi: 10.1099/ijsem.0.000998

Scholz, H. C., Hubalek, Z., Sedlácek, I., Vergnaud, G., Tomaso, H., Al Dahouk, S., et al. (2008b). Brucella microti sp. nov., isolated from the common vole Microtus arvalis. Int. J. Syst. Evol. Microbiol. 58, 375-382. doi: 10.1099/ijs.0.65356-0

Scholz, H. C., Nockler, K., Llner, C. G., Bahn, P., Vergnaud, G., Tomaso, H., et al. (2010). Brucella inopinata sp. nov., isolated from a breast implant infection. Int. J. Syst. Evol. Microbiol. 60, 801-808. doi: 10.1099/ijs.0.011148-0

Scholz, H. C., Pfeffer, M., Witte, A., Neubauer, H., Al Dahouk, S., Wernery, U., et al. (2008a). Specific detection and differentiation of Ochrobactrum anthropi, Ochrobactrum intermedium and Brucella spp. by a multi-primer PCR that targets the recA gene. J. Med. Microbiol. 57:64-71. doi: 10.1099/jmm.0. 47507-0

Shilton, C. M., Brown, G. P., Benedict, S., and Shine, R. (2008). Spinal arthropathy associated with Ochrobactrum anthropi in free-ranging cane toads (Chaunus [Bufo] marinus) in Australia. Vet. Pathol. 45, 85-94. doi: 10.1354/vp.45-1-85

Soler-Lloréns, P., Gil-Ramírez, Y., Zabalza-Baranguá, A., Iriarte, M., CondeÁlvarez, R., Zúñiga-Ripa, A., et al. (2014). Mutants in the lipopolysaccharide of Brucella ovis are attenuated and protect against B. ovis infection in mice. Vet. Res. 45, 1-11. doi: 10.1186/s13567-014-0072-0

Sperandeo, P., Dehò, G., and Polissi, A. (2009). The lipopolysaccharide transport system of Gram-negative bacteria. Biochim. Biophys. Acta 1791, 594-602. doi: 10.1016/j.bbalip.2009.01.011

Terwagne, M., Ferooz, J., Rolán, H. G., Sun, Y.-H., Atluri, V., Xavier, M. N., et al. (2013). Innate immune recognition of flagellin limits systemic persistence of Brucella. Cell. Microbiol. 15, 942-960. doi: 10.1111/cmi.12088

Tiller, R. V., Gee, J. E., Frace, M. A., Taylor, T. K., Setubal, J. C., Hoffmaster, A. R., et al. (2010b). Characterization of novel Brucella strains originating from wild native rodent species in North Queensland, Australia. Appl. Environ. Microbiol. 76, 5837-5845. doi: 10.1128/AEM.00620-10

Tiller, R. V., Gee, J. E., Lonsway, D. R., Gribble, S., Bell, S. C., Jennison, A. V., et al. (2010a). Identification of an unusual Brucella strain (BO2) from a lung biopsy in a 52 year-old patient with chronic destructive pneumonia. BMC Microbiol. 10:23. doi: 10.1186/1471-2180-10-23

Tsai, C. M., and Frasch, C. E. (1982). A sensitive silver stain for detecting lipopolysaccharides in polyacrylamide gels. Anal. Biochem. 119, 115-119. 
Tsolis, R. M., Seshadri, R., Santos, R. L., Sangari, F. J., Lobo, J. M., de Jong, M. F., et al. (2009). Genome degradation in Brucella ovis corresponds with narrowing of its host range and tissue tropism. PLoS ONE 4:e5519. doi: 10.1371/journal.pone.0005519

Vaidya, S. A., Citron, D. M., Fine, M. B., Murakami, G., and Goldstein, E. J. C. (2006). Pelvic abscess due to Ochrobactrum anthropi in an immunocompetent host : case report and review of the literature. J. Clin. Microbiol. 44, 1184-1186. doi: 10.1128/JCM.44.3.1184-1186.2006

Wattam, A. R., Abraham, D., Dalay, O., Disz, T. L., Driscoll, T., Gabbard, J. L., et al. (2014a). PATRIC, the bacterial bioinformatics database and analysis resource. Nucleic Acids Res. 42, 1-11. doi: 10.1093/nar/gkt1099

Wattam, A. R., Foster, J. T., Mane, S. P., Beckstrom-sternberg, S. M., Beckstromsternberg, J. M., Dickerman, A. W., et al. (2014b). Comparative phylogenomics and evolution of the Brucellae reveal a path to virulence. J. Bacteriol. 196, 920-930. doi: 10.1128/JB.01091-13

Wattam, A. R., Inzana, T. J., and Williams, K. P. (2012). Comparative genomics of early-diverging Brucella strains reveals a novel lipolysaccharide biosynthesis pathway. MBio 3, 1-11. doi: 10.1128/mBio.00246-12

Whatmore, A. M., Dale, E.-J., Stubberfield, E., Muchowski, J., Koylass, M., Dawson, C., et al. (2015). Isolation of Brucella from a White's tree frog (Litoria caerulea). JMM Case Reports 2:e000017. doi: 10.1099/jmmcr.0.000017
Whatmore, A. M., Davison, N., Cloeckaert, A., Al Dahouk, S., Zygmunt, M S., Brew, S. D., et al. (2014). Brucella papionis sp. nov., isolated from baboons (Papio spp.). Int. J. Syst. Evol. Microbiol. 64(Pt 12), 4120-4128. doi: 10.1099/ijs.0.065482-0

Zygmunt, M. S., Jacques, I., Bernardet, N., and Cloeckaert, A. (2012). Lipopolysaccharide heterogeneity in the atypical group of novel emerging Brucella species. Clin. Vaccine Immunol. 19, 1370-1373. doi: 10.1128/CVI.00300-12

Conflict of Interest Statement: The authors declare that the research was conducted in the absence of any commercial or financial relationships that could be construed as a potential conflict of interest.

Copyright (C) 2016 Soler-Lloréns, Quance, Lawhon, Stuber, Edwards, Ficht, RobbeAusterman, O'Callaghan and Keriel. This is an open-access article distributed under the terms of the Creative Commons Attribution License (CC BY). The use, distribution or reproduction in other forums is permitted, provided the original author(s) or licensor are credited and that the original publication in this journal is cited, in accordance with accepted academic practice. No use, distribution or reproduction is permitted which does not comply with these terms. 